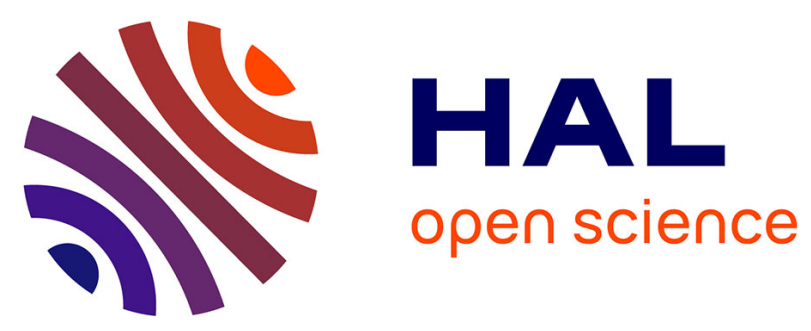

\title{
Nonlinear magnetic vibration absorber for passive control of a multi-storey structure
}

Stefania Lo Feudo, Cyril Touzé, Jean Boisson, Gwendal Cumunel

\section{To cite this version:}

Stefania Lo Feudo, Cyril Touzé, Jean Boisson, Gwendal Cumunel. Nonlinear magnetic vibration absorber for passive control of a multi-storey structure. Journal of Sound and Vibration, 2019, 438, pp.33-353. 10.1016/j.jsv.2018.09.007 . hal-01891645

\section{HAL Id: hal-01891645 \\ https://hal.science/hal-01891645}

Submitted on 9 Oct 2018

HAL is a multi-disciplinary open access archive for the deposit and dissemination of scientific research documents, whether they are published or not. The documents may come from teaching and research institutions in France or abroad, or from public or private research centers.
L'archive ouverte pluridisciplinaire HAL, est destinée au dépôt et à la diffusion de documents scientifiques de niveau recherche, publiés ou non, émanant des établissements d'enseignement et de recherche français ou étrangers, des laboratoires publics ou privés. 


\title{
Nonlinear magnetic vibration absorber for passive control of a multi-storey structure
}

\author{
S. Lo Feudo ${ }^{\mathrm{a}, \mathrm{b}, *}$, C. Touzé ${ }^{\mathrm{b}}$, J. Boisson $^{\mathrm{b}}$, G. Cumunel $^{\mathrm{c}}$ \\ ${ }^{a}$ QUARTZ (EA7393), VAST, Supméca - ISMEP, Saint-Ouen, France \\ ${ }^{b} I M S I A$, ENSTA ParisTech-CNRS-EDF-CEA, Université Paris-Saclay, Palaiseau, France \\ ${ }^{c}$ Université Paris-Est, Laboratoire Navier (UMR 8205), CNRS, ENPC, IFSTTAR, F-77455 Marne-la-Vallée, France
}

\begin{abstract}
A nonlinear magnetic vibration absorber is presented and used to control vibration of a three-storey structure. A distinctive feature of the absorber concerns its versatility for tuning the linear and nonlinear stiffness coefficients, depending on simple geometric design parameters such as the distance between fixed magnets and the moving one. In particular, the absorber can be set either as a nonlinear tuned vibration absorber, a nonlinear energy sink, or a bistable tuned vibration absorber, according to whether the linear stiffness term is positive, vanishing, or negative. The response of the primary structure and the vibration mitigation are investigated in the cases of impulsive shock, free vibration with imposed initial displacement, and single frequency excitation. Significant reductions of the primary structure vibrations are obtained for the three cases investigated, showing the ability of using a vibration absorber only relying on magnetic forces for passive control. The detailed comparisons of the absorbers performance show that, in this case study, no general guidelines can be easily deduced for selecting one of the three tunings for a nonlinear absorber. Depending on the excitation, the vibratory levels, and the frequency content of the excitation, the three configurations show advantages and drawbacks that are discussed.
\end{abstract}

Keywords: Passive control, Nonlinear absorber, NES, Magnetic Vibration Absorber, vibration experiments

\section{Introduction}

In the civil engineering field, vibration control devices are ever more required to face dynamic excitation such as wind load and ground motion acceleration. Several studies and applications concern the Tuned Mass Damper (TMD), which consists in a linear device added to a primary structure and tuned to one resonant mode. Early studies were conducted in [1-3], and the main parameter optimisation criteria are based on the minimization either of the transfer function maximum or of the energy $[4,5]$, as well as on the pole location $[6,7]$. The effectiveness of one or multiple TMD added on single or multi degree of freedom systems subjected to harmonic, random, and seismic excitations has been studied in [8-12]. Among the experimental devices, recent studies deal with TMD based on the eddy currents damping effect, [13-17].

The performance of the TMD is very effective in the case of linear systems and has been proven to be a very reliable passive mitigation device in a large number of contexts. However it is known to bear some

\footnotetext{
* Corresponding author

Email addresses: stefania.lofeudo@supmeca.fr (S. Lo Feudo), cyril.touze@ensta-paristech.fr (C. Touzé), jean.boisson@ensta-paristech.fr (J. Boisson), gwendal.cumunel@enpc.fr (G. Cumunel)
} 
limitations, as e.g. its tuning to only one resonant frequency and its sensitivity to the primary structure uncertainty [18-20]. To overcome these drawbacks, the use of nonlinear vibration absorbers is studied as an alternative to the TMD in order to enhance the range of effectiveness in terms of frequency or vibration amplitude.

A first idea is to use an essential nonlinearity, with no linear restoring force, to design the vibration absorber. This leads to the so-called NES for Nonlinear Energy Sink [21-23], which has no eigenfrequency and can tune to any frequency content displayed by the primary vibrating structure, thus extending the typical frequency bandwidth of interest [21, 23, 24]. In particular, it has been demonstrated that, above a certain force threshold, an irreversible energy transfer from the main system to the NES is activated [23, 25]. This phenomenon has been named Targeted Energy Transfer (TET), and it has also been shown that the activation threshold increases with frequency [26]. Along the same lines, recent studies have considered the case of a negative linear stiffness to create a Bistable Nonlinear Tuned Vibration Absorber (BNTVA) as an extension to the NES. In particular, it has been shown for this configuration that the minimal energy required to activate the energy transfer is reduced [27-29]. Moreover, above a certain load level, bistability leads to quasiperiodic or chaotic nonlinear oscillations which give rise to a better damping capacity, as reported theoretically and experimentally [26, 30,31]. An exhaustive literature review including the methods of analysis and the bistable experimental devices used for the energy harvesting is reported in [32].

Another approach consists in using the nonlinearity to extend the range of optimal control in terms of vibration amplitudes. This idea leads to the concept of the nonlinear tuned vibration absorber (NLTVA), wherein the efficacy of the absorber is increased to nonlinear vibration range, with oscillation frequency depending on amplitude. In the late 80's, Rice and McCraith proposed a nonlinear vibration absorber with cubic stiffness for the vibration control of a linear oscillator [33]. Jordanov and Cheshankov carried out a multi-criteria optimization for the absorber design [34], whereas Shaw et al. and Natsiavas focused on the stability of the solutions of two coupled nonlinear oscillators [35, 36]. More recently, a numerical study was conducted by Alexander and Schilder on a nonlinear absorber, [37], composed of two sets of perpendicular springs generating a hardening stiffness, and attached to the top floor of a Multi Degree of Freedom (MDOF) building structure subjected to seismic ground motion. The absorber parameters are optimized by minimizing the $L_{2}-$ norm and its performance is compared to that of a linear TMD. As a result, the authors found dangerous detached resonance curves, occurring in the frequency response of the primary structure for vanishing linear stiffness. Finally, for their case study, they concluded that the TMD performs better than a nonlinear absorber. Closed detached resonance curve were also measured experimentally by Gatti et al. [38], who tested a two degree of freedom system composed of an electro-dynamic shaker and a nonlinear Duffing oscillator of smaller mass. More recently, the rule given by Den Hartog to design the TMD parameters and known as the "equal-peak method" has been extended to the NLTVA, giving rise to a number of studies and methods for vibration and flutter mitigation [39-44], showing the efficiency of the NLTVA in a number of contexts.

Concerning the experimental realization of nonlinear vibration absorbers, numerous studies used mechanical devices to create the needed nonlinear restoring force: wires without tension to create a NES [45], vibro-impact NES [46-50], magnetic-strung absorbers [51], conical springs [52], beams with variable section for designing an NLTVA [53], and piezoelectric devices [54]. The idea of using magnetic forces has been investigated by Jo and Yabuno [55], who studied an experimental nonlinear vibration absorber made of repulsive magnets whose natural frequency is twice that of the principal system. Electromagnets and permanent magnets have also been used in $[56,57]$ to adjust the nonlinear stiffness by simply varying the magnets gap.

This study is set within the context of absorbers obtained thanks to nonlinear magnetic forces. More specifically, the Magnetic Vibration Absorber (MVA) designed in [31] is used here for the passive control of a multi-storey structure. The nonlinear absorber is composed of a moving magnet placed within a magnetic field induced by fixed magnets. Thanks to simple geometric adjustments, either a positive, vanishing, or negative linear stiffness can be obtained, so that the present device can be tuned as NLTVA, NES, or BNTVA. The goal of this paper is to present the effectiveness of this versatile vibration damper as well as to compare the performance of each configuration on the same experimental set-up. The paper is organized as follows. In Section 2, the experimental MVA and its theoretical formulation is presented. Section 3 presents 


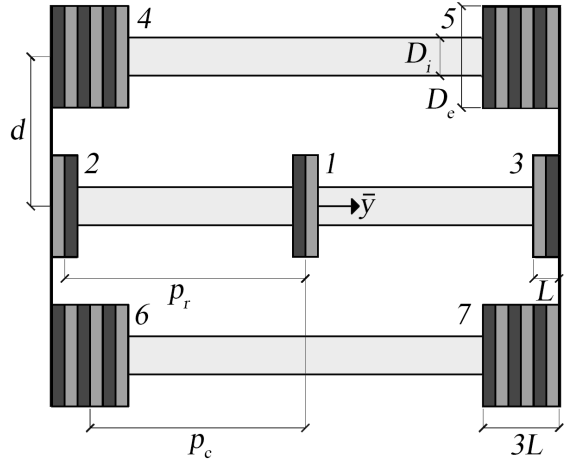

(a)

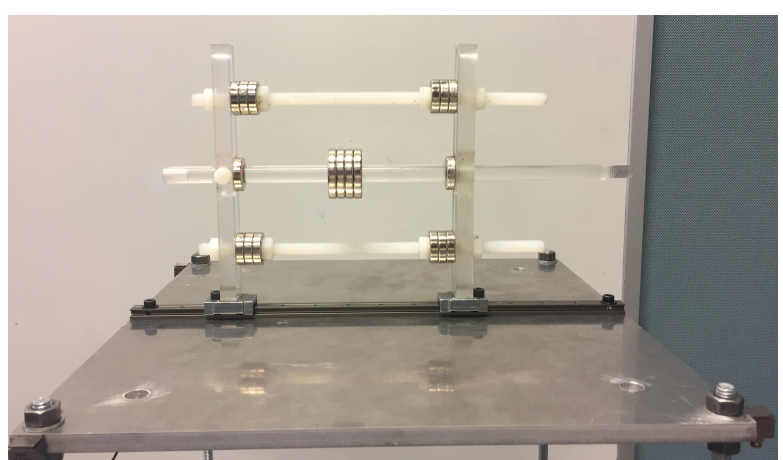

(b)

Figure 1. (a) Schematic representation of the MVA, composed of a moving magnet 1 surrounded by fixed magnets 2 to 7 . The lengths $d, p_{r}$, and $p_{c}$ are adjustable so as to set the linear and nonlinear magnetic stiffnesses exerted on magnet 1 . (b) Picture of the MVA used in the experiments and located on the top floor of the three-storey structure.

the experimental MDOF structure and the identification procedure used to obtain its modal parameters. Then, in Section 4 the response of the coupled system composed of the structure and the MVA is studied for various load cases. Finally, some conclusions are drawn in Section 5.

\section{Nonlinear Magnetic Vibration Absorber}

The Magnetic Vibration Absorber (MVA) was introduced first in [31] and its schematic representation is shown in Fig. 1(a). This vibration absorber relies on magnetic forces only and different configurations can be easily obtained thanks to the adjustment of the geometric parameters (i.e. the distance and angles between magnets), allowing one to set the linear and nonlinear stiffness coefficients of the MVA. The MVA is composed of a moving magnet (numbered 1 in Fig. 1(a)) which can move along a polymeric rod. This moving element can be composed of one or more magnets located in the middle of two repulsive magnets with inverted polarity (numbered 2 and 3 in Fig. 1(a)) and distant of a length $2 p_{r}$. Other magnets (numbered 4 to 7 ), having the same polarity as magnet 1 and thus being attractive, are hosted on two parallel rods on each side of the moving element rod. The setting of their lateral and longitudinal locations, $d$ and $p_{c}$ respectively, modifies the magnetic field experienced by the moving element and they are called corrective magnets. In the next subsection, the theoretical model obtained from the multipolar development derived in [31] is first recalled. Then, the stiffness values reachable with the device used for the experiments presented in the paper are detailed in order to give an overview of the setting of the MVA.

\subsection{Theoretical model}

The interaction energy between the magnets composing the MVA leads to either attractive or repulsive nonlinear forces between the fixed magnets and the moving one. In the present case, the magnetic field induced by the magnets has been estimated by a multipolar decomposition, giving a better approximation of the magnetic field than the dipolar model. More precisely, multipolar developments are used in [31] for magnets 2 to 7 , whereas the moving magnet 1 was simply modeled as a dipole. In the present contribution, this formulation is improved by taking into account the specific ring geometry of magnet 1 . The full analytic developments of this improvement is detailed in Appendix A.

The total force acting on the moving magnet is given by

$$
\begin{aligned}
F_{T o t} & \approx-\left(K_{1}^{r}\left(p_{r}\right)-K_{1}^{c}\left(p_{c}, d\right)\right) \bar{y}-\left(K_{3}^{r}\left(p_{r}\right)-K_{3}^{c}\left(p_{c}, d\right)\right) \bar{y}^{3}-\left(K_{5}^{r}\left(p_{r}\right)-K_{5}^{c}\left(p_{c}, d\right)\right) \bar{y}^{5} \\
& \approx-K_{1}\left(p_{r}, p_{c}, d\right) \bar{y}-K_{3}\left(p_{r}, p_{c}, d\right) \bar{y}^{3}-K_{5}\left(p_{r}, p_{c}, d\right) \bar{y}^{5} .
\end{aligned}
$$

where the superscripts $r$ and $c$ stand for repulsive and corrective respectively, $p_{r}$ and $p_{c}$ are the distances along the $\bar{y}$-axis between the moving magnet and the repulsive and corrective magnets respectively, and $d$ is the transverse distance between the repulsive and the corrective magnets. 
Therefore, the total force $F_{T o t}$ depends on a linear stiffness coefficient, $K_{1}$, and nonlinear cubic and quintic stiffness coefficients, $K_{3}$ and $K_{5}$ respectively. Their expressions are recalled in Appendix A. It should be noted that the nonlinear stiffness terms depend on the distances between the magnets, which can be easily tuned for the MVA experimental device.

\subsection{Experimental device}

The MVA device has been designed and built to meet the requirements for the vibration mitigation of a three-storey structure. One of the advantage of the MVA is that its moving mass can be easily increased or decreased according to design needs. Consequently, in the present study, the design of the MVA differs from that used in [31] since the primary structure is a three-storey structure with an important mass. In particular, four magnets, the dimensions of which differ from the fixed magnets, are used for the moving element, as shown in Fig. 1(b). Table 1 summarizes the selected values for designing the MVA, where $m_{M V A}$ denotes the mass of one ring magnet, $\mathcal{M}$ the magnetic moment, $D_{e}$ and $D_{i}$ the external and internal diameters respectively, $L$ the thickness, and $n$ the total number of magnets used. More precisely, corrective magnets are split into four groups, each one made up of three permanent magnets.

Once the properties of the magnets are chosen, the distances $p_{r}$ and $d$ can be set manually in order to tune the stiffness values needed to damp vibrations of the primary structure. In our case, the distance $p_{c}$ is such that $p_{c}=p_{r}-L$ so that setting the length $p_{r}$ determines $p_{c}$. Figs. 2(a), (c), and (e) show the evolution of the stiffness coefficients $K_{1}, K_{3}$, and $K_{5}$ calculated as a function of the distance $p_{r}$, for different values of $d$ easily attainable with the design parameters reported in Table 1 . These theoretical curves can be used as a design tool to predict the geometry of the MVA to be set in order to obtain either a NLTVA, a NES, or a BNTVA. One can observe that large values of the coefficient $K_{1}$ can be obtained with small values of $p_{r}$, whereas negative values of $K_{1}$, needed to achieve a BNTVA, are accessible for large values of $p_{r}$. Otherwise, the nonlinear stiffness coefficients $K_{3}$ and $K_{5}$ become negative only when magnets are close.

In the experiments, the primary structure is a multi-storey structure with its first resonance frequency measured at $7.1 \mathrm{~Hz}$, as detailed in the next section. Using the theoretical results depicted in Fig. 2(a), (c), and (e), different configurations of the MVA are chosen to meet the requirements to design a NLTVA, a NES, or a BNTVA. In particular, three different configurations are considered for each case of absorber:

- For the NLTVA, the main design rule is to select the frequency of the magnetic oscillator in the vicinity of the first resonance frequency of the primary structure. The three selected designs respect this requirement, leading to three different values of the nonlinear stiffness coefficients. However, as explained in [31], the selection of the nonlinear stiffness coefficients is not fully independent from that of the linear one.

- For the NES, three configurations with an almost vanishing linear stiffness have been selected.

- For the BNTVA, the negative linear stiffness has been chosen such that the equivalent oscillation frequency has the same value for the three cases considered.

Table 2 summarizes the possible values of $p_{r}$ and $d$, together with the resulting values of the linear and nonlinear stiffness coefficients obtained with the multipolar model for these designs. For four configurations, the oscillator frequency is not specified because the selected coefficients lead to a negative linear stiffness

\begin{tabular}{|c|cccccc|}
\hline Magnets & $m_{M V A}[\mathrm{~kg}]$ & $D_{e}[\mathrm{~mm}]$ & $D_{i}[\mathrm{~mm}]$ & $L[\mathrm{~mm}]$ & $n$ & $\mathcal{M}\left[\mathrm{Am}^{2}\right]$ \\
\hline Moving (1) & 0.023 & 30 & 10 & 5 & 4 & 2.88 \\
Repulsive (2,3) & 0.010 & 20 & 10 & 5 & 2 & 2.43 \\
Corrective (4-7) & 0.010 & 20 & 10 & 5 & 12 & 1.87 \\
\hline
\end{tabular}

Table 1. Properties of the magnets composing the MVA designed for the experimental set-up shown in Fig. 1(b). 
$K_{1}$. The corresponding nonlinear restoring forces evaluated according to Eq. (1) are depicted in Figs. 2(b), (d), and (f).

In the following, the experiments are conducted with these nine configurations in order to analyze the influence of the nonlinear coefficients on the dynamical behaviour of the coupled system.

\section{Multi-storey structure}

In this section, a detailed analysis of the primary structure, the multi-storey structure shown in Fig. 3, is carried out. Particular emphasis is given on the modal identification in order to have a complete view of its vibrational behaviour. Note that using such a magnetic absorber on a full scale building might be difficult, leading to numerous difficulties such as creating strong enough magnetic fields, or managing interference with electrical systems inside the building. However, the application to a real-life situation is not the concern of this study, where the multi-storey building is used as a multi degree of freedom test structure chosen for the ease of placing the MVA at its top and running experimental tests.

\subsection{Experimental setup}

The primary structure is a three-storey structure composed of horizontal aluminum plates linked together by four threaded rods. The MVA is fixed on the top floor along one of the principal axis of the structure. The mass ratio between the damper and the principal structure, which also takes into account the support frame of the MVA, is about $1.3 \%$. Note that, in order to uncouple the vibrations of the structure along the $y$-axis and its orthogonal direction, two cross bracings have been added on the opposite sides of the second floor.

The dynamic behaviour of the structure alone is investigated by locking the MVA moving mass, whereas the effect of the absorber on the vibration of the primary structure is studied by letting the moving magnet free to oscillate. In order to achieve forced vibration tests along the $y$-axis, the structure is fixed to a sliding stage connected to a Brüel \& Kjaer shaker Type 4808. Hammer impact tests are also conducted to obtain free vibration measurements. For these tests, the sliding stage is locked. The acceleration of each floor is measured by two accelerometers Brüel \& Kjaer Type 4507-B-004, located near the plates wedge. The displacement of the MVA moving mass is measured by means of a non-contact optical sensor Keyence LS-7070M.

\begin{tabular}{|c|cc|ccc|c|}
\hline NLTVA & $p_{r}[\mathrm{~mm}]$ & $d[\mathrm{~mm}]$ & $K_{1}[\mathrm{~N} / \mathrm{m}]$ & $K_{3}\left[\mathrm{~N} / \mathrm{m}^{3}\right]$ & $K_{5}\left[\mathrm{~N} / \mathrm{m}^{5}\right]$ & $f_{M V A}[\mathrm{~Hz}]$ \\
\hline$I$ & 64 & 48 & $1.80 \cdot 10^{2}$ & $1.24 \cdot 10^{5}$ & $7.98 \cdot 10^{7}$ & 7.05 \\
$I I$ & 61 & 39 & $1.82 \cdot 10^{2}$ & $3.89 \cdot 10^{5}$ & $1.99 \cdot 10^{8}$ & 7.07 \\
$I I I$ & 51 & 28 & $1.83 \cdot 10^{2}$ & $1.44 \cdot 10^{6}$ & $1.59 \cdot 10^{9}$ & 7.09 \\
\hline NES & $p_{r}[\mathrm{~mm}]$ & $d[\mathrm{~mm}]$ & $K_{1}[\mathrm{~N} / \mathrm{m}]$ & $K_{3}\left[\mathrm{~N} / \mathrm{m}^{3}\right]$ & $K_{5}\left[\mathrm{~N} / \mathrm{m}^{5}\right]$ & $f_{M V A}[\mathrm{~Hz}]$ \\
\hline$I$ & 68 & 33 & $9.87 \cdot 10^{-1}$ & $1.65 \cdot 10^{5}$ & $1.32 \cdot 10^{8}$ & 0.52 \\
$I I$ & 61 & 30 & $1.07 \cdot 10^{0}$ & $3.60 \cdot 10^{5}$ & $3.55 \cdot 10^{8}$ & 0.54 \\
$I I I$ & 54 & 27 & $-2.93 \cdot 10^{-1}$ & $8.62 \cdot 10^{5}$ & $1.08 \cdot 10^{9}$ & - \\
\hline BNTVA & $p_{r}[\mathrm{~mm}]$ & $d[\mathrm{~mm}]$ & $K_{1}[\mathrm{~N} / \mathrm{m}]$ & $K_{3}\left[\mathrm{~N} / \mathrm{m}^{3}\right]$ & $K_{5}\left[\mathrm{~N} / \mathrm{m}^{5}\right]$ & $f_{M V A}[\mathrm{~Hz}]$ \\
\hline$I$ & 68 & 28 & $-8.55 \cdot 10^{1}$ & $9.28 \cdot 10^{4}$ & $1.31 \cdot 10^{8}$ & - \\
$I I$ & 59 & 27 & $-8.31 \cdot 10^{1}$ & $3.78 \cdot 10^{5}$ & $5.00 \cdot 10^{8}$ & - \\
$I I I$ & 55 & 26 & $-8.51 \cdot 10^{1}$ & $6.68 \cdot 10^{5}$ & $9.46 \cdot 10^{8}$ & - \\
\hline
\end{tabular}

Table 2. Geometry settings of the MVA used in the experiments, together with the theoretical stiffness coefficients and frequencies resulting from the multipolar model. 


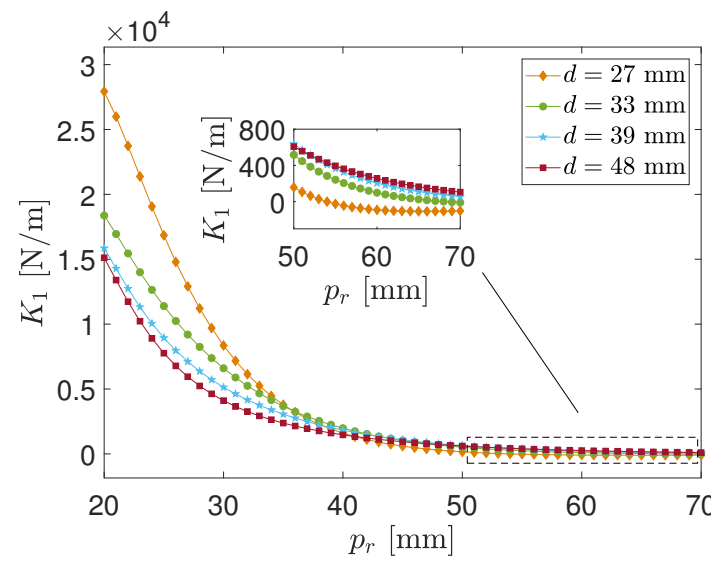

(a)

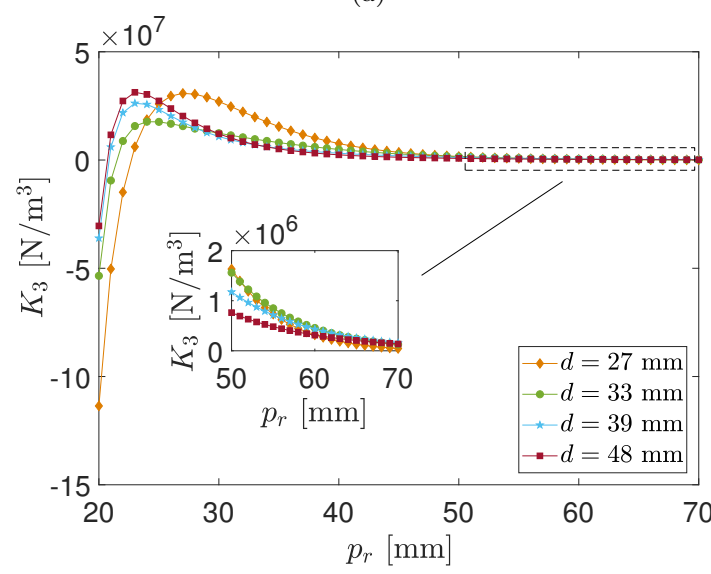

(c)

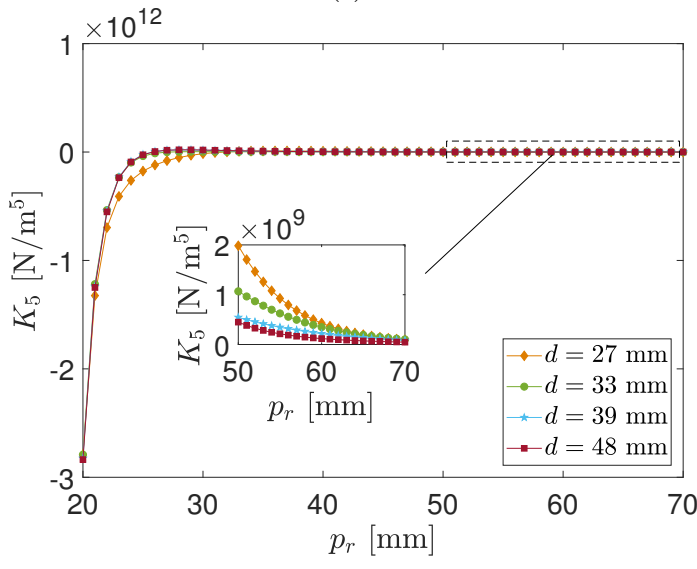

(e)

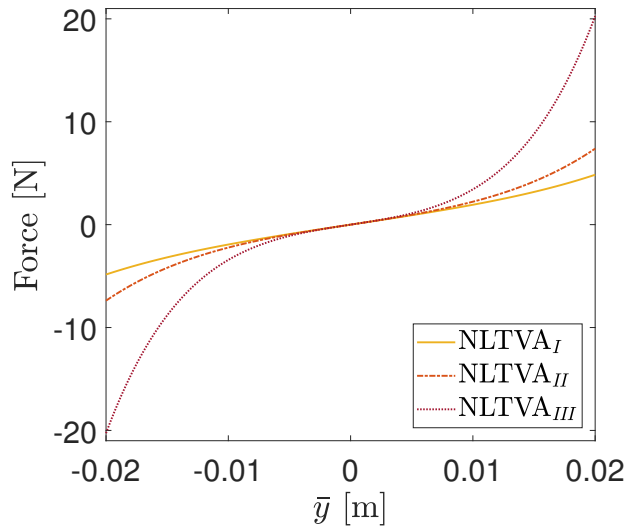

(b)

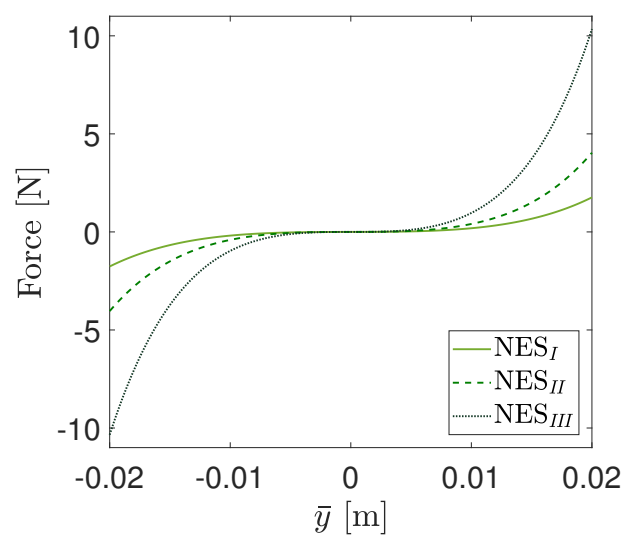

(d)

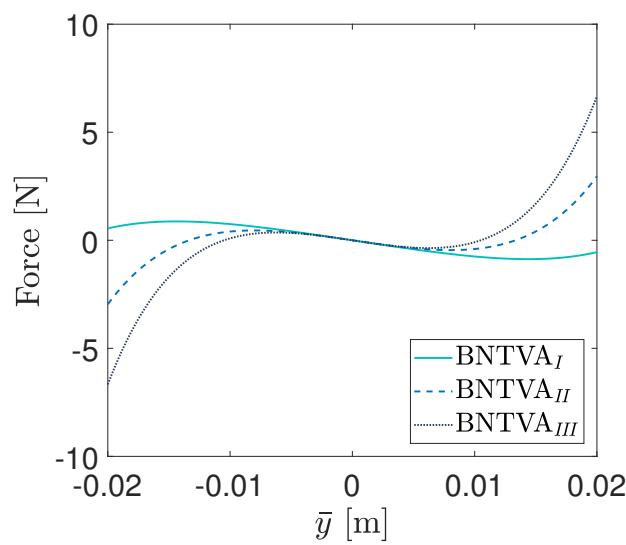

(f)

Figure 2. MVA theoretical stiffness coefficients as a function of the distance $p_{r}$ for different values of the distance $d$ (the distance $p_{c}$ is such that $p_{c}=p_{r}-L$ ): (a) linear stiffness coefficient $K_{1}$, (c) cubic stiffness coefficient $K_{3}$, and (e) quintic stiffness coefficient $K_{5}$. Nonlinear total force for several MVA configurations (Table 2) : (b) NLTVA, (d) NES, and (f) BNTVA. 


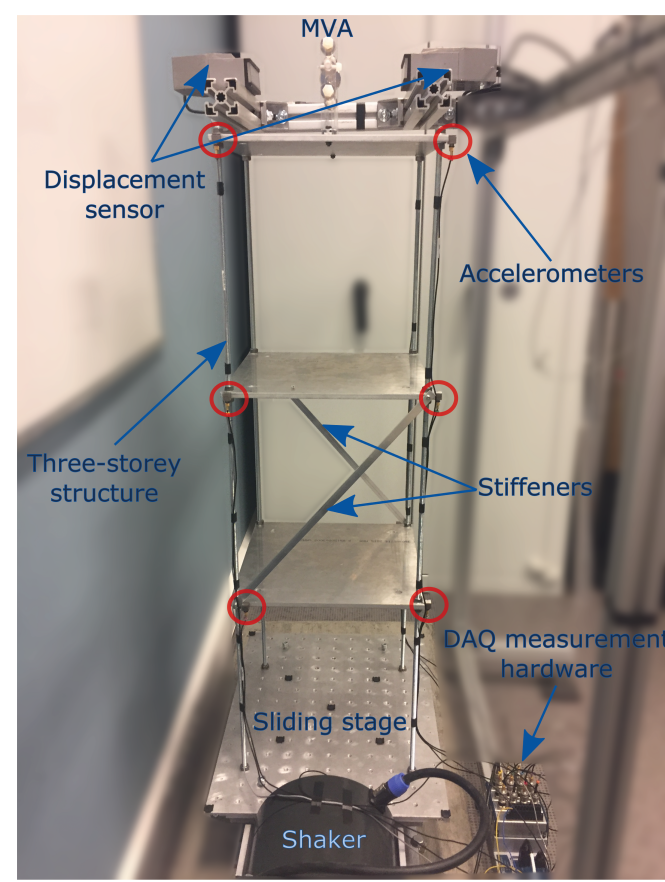

(a)

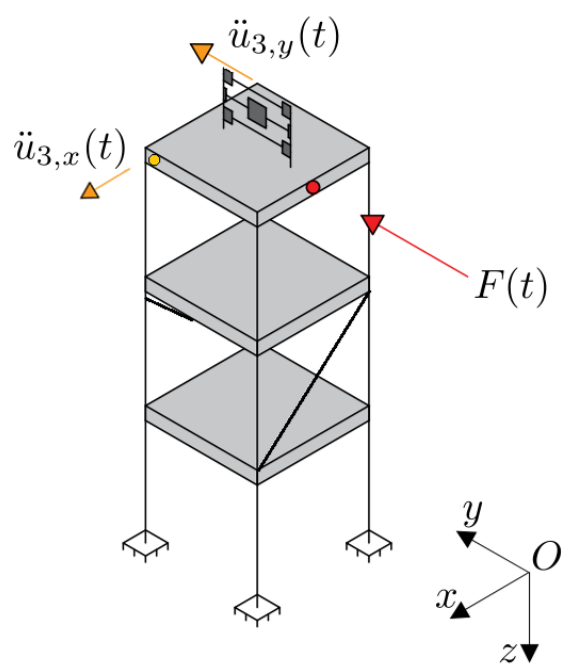

(b)

Figure 3. (a) Experimental multi-storey structure coupled with the MVA located at the top floor. (b) Scheme of the coupled system in the case of impulsive excitation, with the shock direction and the accelerometers location at the top floor.

\subsection{Modal identification}

The modal parameters of the structure are evaluated through impact hammer tests, whose force direction and impact location are shown in Fig. 3(b). It is worth noting that, although the impact is given only in the $y$-direction, experimental results show that smaller amplitude vibrations are also measured in the orthogonal $x$-direction.

Fig. 4 presents the main results of the modal analysis, i.e. the Frequency Response Function (FRF) of the last floor and the mode shapes of the structure in the $y$-direction. The frequencies of the first three modes in the $x$ and $y$ directions can be easily identified from the FRF, as well as torsional modes, for which a unidirectional shock gives peaks of almost equal amplitude in the FRF curves obtained for both directions. Then, a continuous wavelet transform method is applied to the experimental measurement signals for the modal identification. Indeed, this method allows an accurate estimation of mode shapes of a MDOF system from its free decay response. For further details on the method, one can see [58]. The mean values and standard deviations, $\sigma_{f_{i}}$ and $\sigma_{\xi_{i}}$ of the modal frequencies $f_{i}$ and damping ratios $\xi_{i}$ for three experimental tests are reported in Table 3.

\section{Analysis of the MVA performance}

In this section, the behaviour of the coupled system composed of the multi-storey structure and the unlocked MVA is studied. First, impact hammer tests are conducted for each of the nine MVA configurations presented in Table 2 in order to assess their vibration mitigation. Performance criteria are then introduced, both in time and frequency domains, in order to obtain a quantitative comparison of their respective performances. The design parameters giving the best performance for each tested case (NLTVA, NES, and BNTVA) are then selected, so that only three configurations will be used in the next experiments. In the last section, the performances of these three configurations are further investigated for the cases of free vibrations with imposed initial displacement and single frequency excitation. 


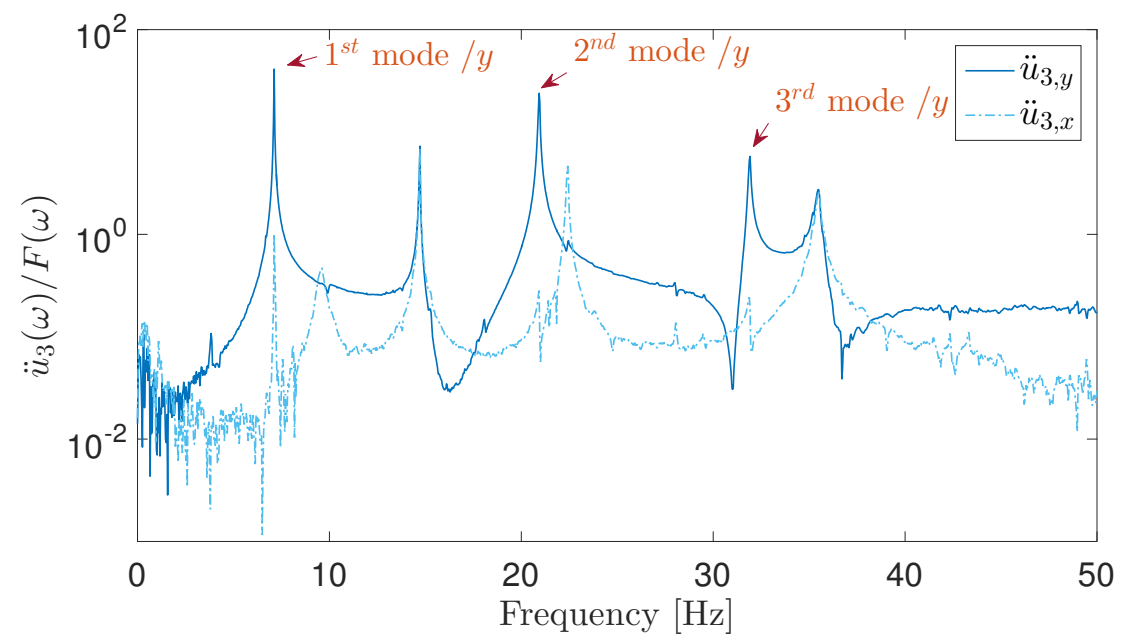

(a)

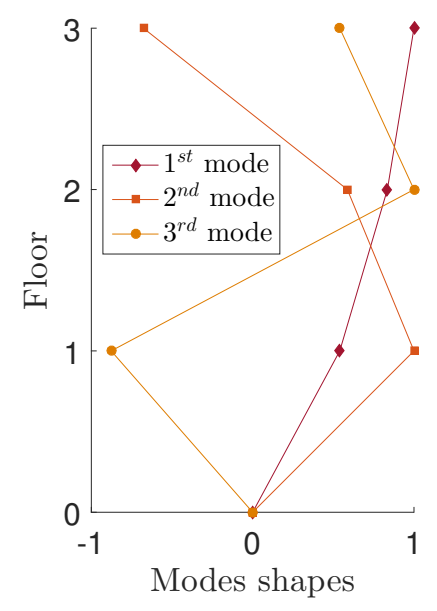

(b)

Figure 4. (a) Experimental FRF of the top floor of the multi-storey structure in both perpendicular directions in the case of shock when the MVA is locked $(F=217.5 \mathrm{~N})$. (b) Mode shapes of the multi-storey structure in the $y$-direction.

\subsection{Impulse response}

The free vibrations of the multi-storey structure coupled with the MVA are measured when the MVA is set as NLTVA, NES, and BNTVA, according to Table 2. To illustrate some typical results, the vibratory behaviour of the structure is compared for the MVA set as NLTVA $_{I I}$ when the MVA moving mass is locked or not. Fig. 5 shows the acceleration measurement signals and the FRF of the top floor in the $y$-direction. As one can observe, the nonlinear vibration absorber mitigates vibrations by decreasing the amplitude of the acceleration signal. In the frequency domain, it results in an attenuation of $5.3 \mathrm{~dB}$ for the first resonant frequency in the $y$-direction.

As a matter of fact, the force amplitude and the resulting displacement of the structure are not the same for all the free vibration tests. Since the results obtained for the structure with the MVA locked and with the different configurations of the MVA have to be compared, the influence of the force amplitude is studied. Figure 6 depicts the top floor displacement normalized to its maximum value, for different shock amplitudes for the case of $\mathrm{NLTVA}_{I I}$. More precisely, the envelope curves shown in Fig. 6 and hereafter have been obtained from the analytic signals of the experimental data, which were evaluated by using the Hilbert's transform. As it can be seen, the experimental curves are almost superposed. This means that the oscillation amplitude of the top floor is almost proportional to the given shock within this range of excitation. The results are similar for all the other configurations of the MVA. For this reason, the normalized oscillations of the top floor will be considered in the next Section in order to compare the response of the primary system

\begin{tabular}{|c|cccc|}
\hline & $f_{i}[\mathrm{~Hz}]$ & $\xi_{i}[\%]$ & $\sigma_{f_{i}}[\mathrm{~Hz}]$ & $\sigma_{\xi_{i}}[\%]$ \\
\hline \multirow{3}{*}{$y$-direction } & 7.1 & 0.2 & $3.7 \cdot 10^{-3}$ & $2.7 \cdot 10^{-4}$ \\
& 20.9 & 0.2 & $1.2 \cdot 10^{-2}$ & $1.3 \cdot 10^{-4}$ \\
& 31.9 & 0.2 & $8.2 \cdot 10^{-3}$ & $1.1 \cdot 10^{-4}$ \\
\hline \multirow{2}{*}{$x$-direction } & 9.6 & 1.9 & $3.5 \cdot 10^{-2}$ & $1.3 \cdot 10^{-3}$ \\
& 22.4 & 0.2 & $1.2 \cdot 10^{-2}$ & $2.5 \cdot 10^{-4}$ \\
\hline \multirow{2}{*}{ Torsional } & 14.7 & 0.1 & $8.2 \cdot 10^{-3}$ & $3.1 \cdot 10^{-4}$ \\
& 35.5 & 0.3 & $2.1 \cdot 10^{-2}$ & $2.9 \cdot 10^{-4}$ \\
\hline
\end{tabular}

Table 3. Modal frequencies and damping ratios of the multi-storey structure and their standard deviations. 


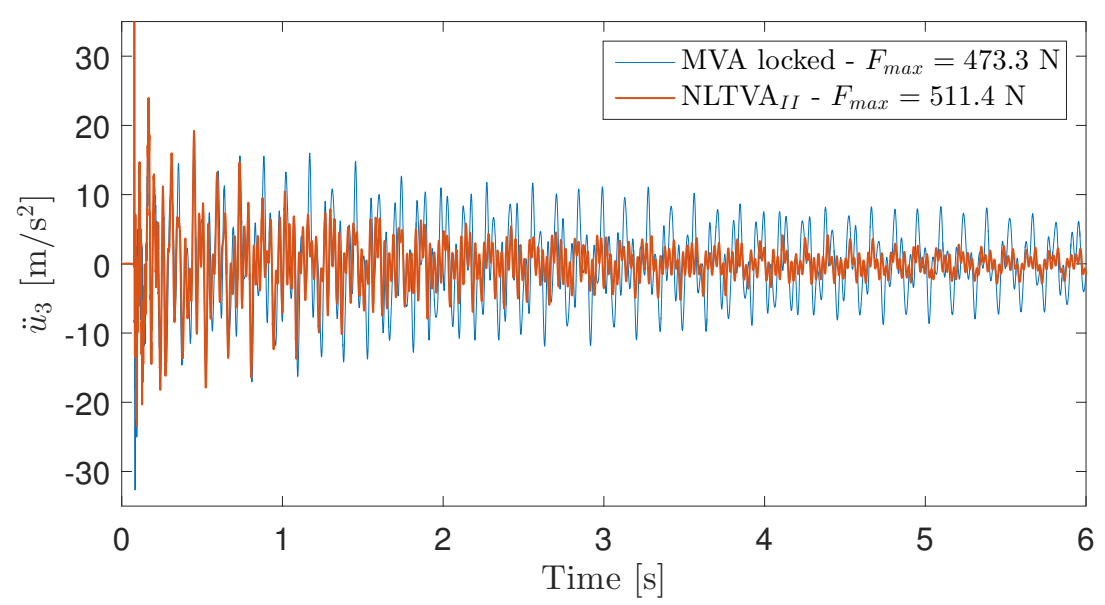

(a)

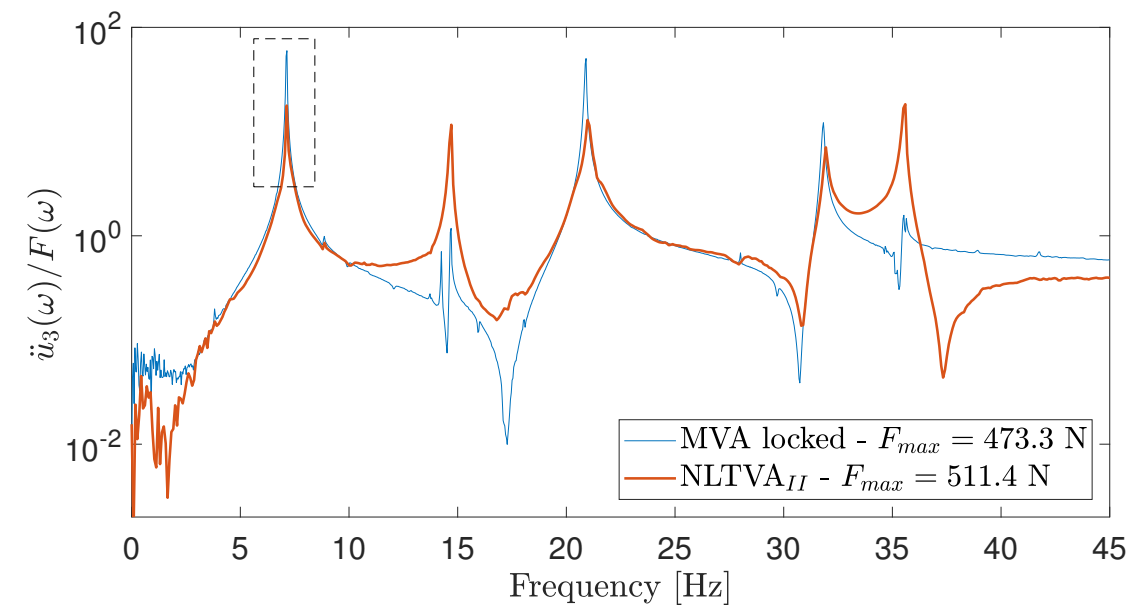

(b)

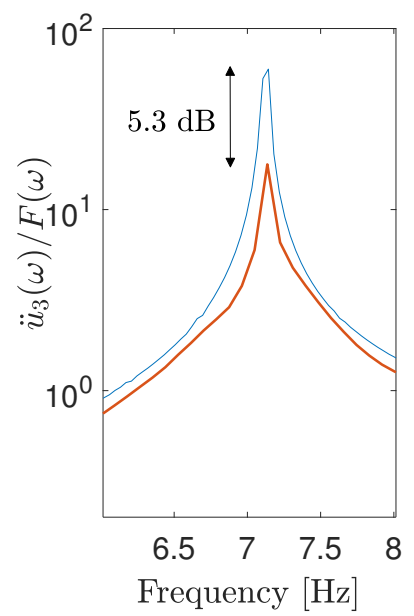

(c)

Figure 5. (a) Acceleration measurement signals of the top floor, (b) FRF obtained in the case of shock when the moving mass of the MVA is locked and when the structure is coupled with the NLTVA $I$, (c) magnification of the FRF first peak.

in the controlled and uncontrolled cases. The curves represented in Fig. 6 also show the edge effect at the beginning of the free vibration displacement signals, which is due to the double integration performed on the experimental acceleration signals.

Next, the behaviour of the coupled system is studied by varying the MVA properties. The results presented hereafter refer to the system response at the top floor in the $y$-direction. For sake of clarity, only the envelope of the displacement signal is presented when the multi-storey structure is coupled with the unlocked MVA. Therefore, the top floor normalized displacement is depicted in Fig. 7(a) when the structure is coupled with the NLTVA for the three different configurations, whereas Fig. 7(b) shows the corresponding MVA displacements. In all cases, the NLTVA allows to reduce the structure vibrations with respect to the case of locked MVA, and the number of the MVA oscillations decreases when the nonlinear coefficients of the MVA are increased. As shown in Fig. 8(a), the NES configurations are also able to reduce the multistorey structure vibrations. However, the oscillations of the NES, reported in Fig. 8(b), seem to be only slightly affected by the nonlinear coefficient variations. This may be due to the fact that, according to experimental measurements, a strong nonlinear effect only appears beyond a magnet displacement of about $5 \mathrm{~mm}$. Finally, the structure and the BNTVA displacements are shown in Fig. 9, where one of the two 


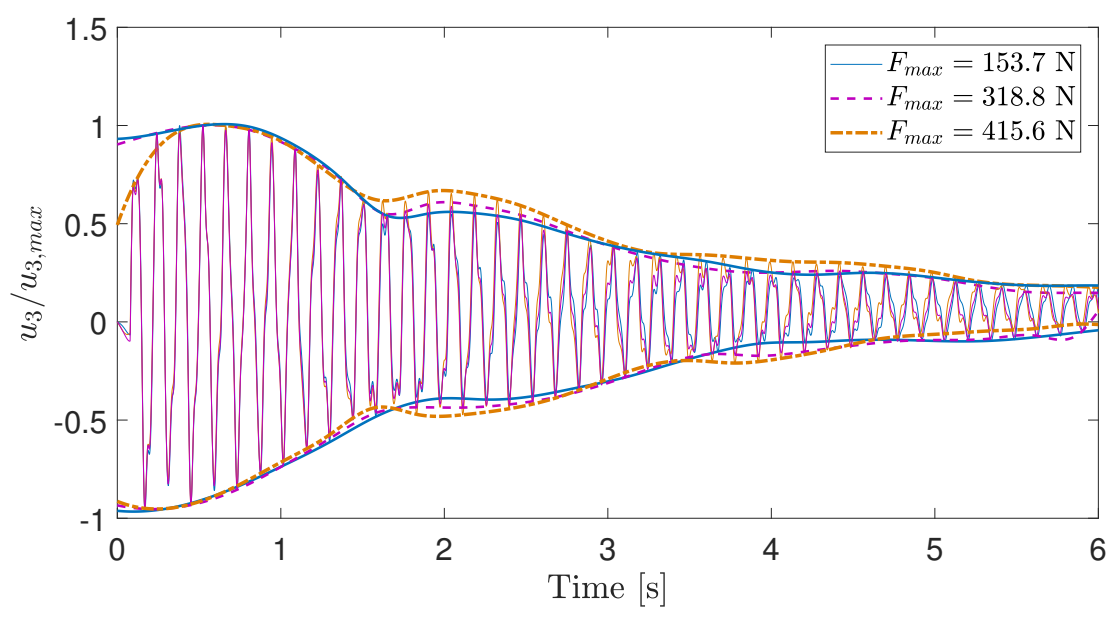

Figure 6. Normalized displacement of the top floor, obtained by double integration of the acceleration measurement signal, in the case of shock when the structure is coupled with the NLTVA $I I$.

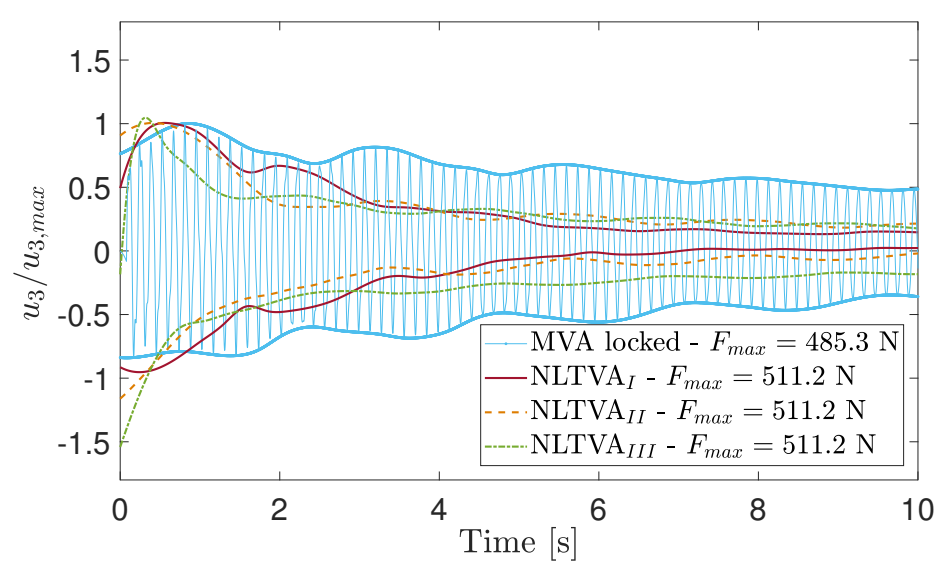

(a)
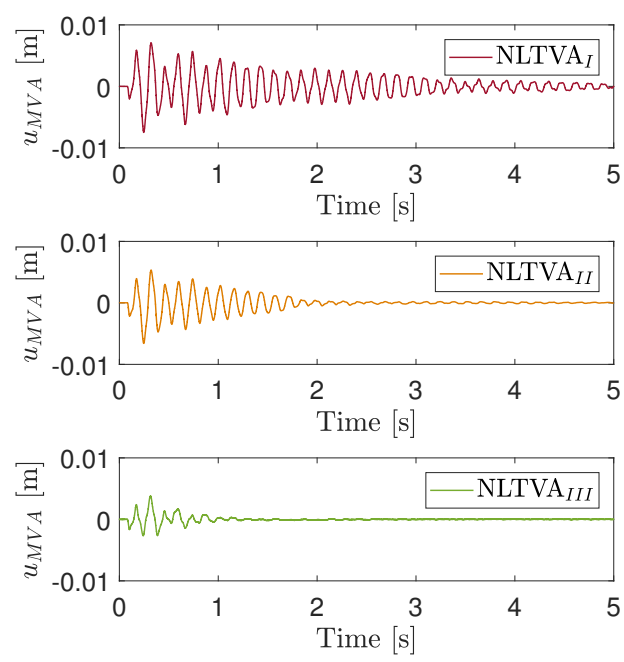

(b)

Figure 7. (a) Normalized displacement of the top floor obtained in the case of shock when the structure is coupled with the different NLTVA configurations, (b) NLTVA displacement.

stable positions was selected as initial conditions of the BNTVA, so that the initial conditions are the same for all experiments. It is worth noting that in this case the zero position presented in Fig. 9(b) corresponds to the aforementioned stable point rather than the MVA mid-point at $\bar{y}=0$. The BNTVA appears to be very effective in terms of vibration damping, as an efficient amplitude decay is obtained. In particular, the tuning of the nonlinear stiffness coefficients gives rise to very different behaviours, with a more effective case for the first configuration, whereas large amplitude modulations are present for the second one.

\subsection{Performance criteria}

The adopted performance criteria are aimed at comparing vibration energy in the frequency and temporal domains, when either the multi-storey structure vibrations are not damped (locked MVA) or passively damped (unlocked MVA). The criteria in the frequency domain, $C_{\bar{f}}, C_{f_{1}}, C_{f_{2}}$, and $C_{f_{3}}$, are defined as: 


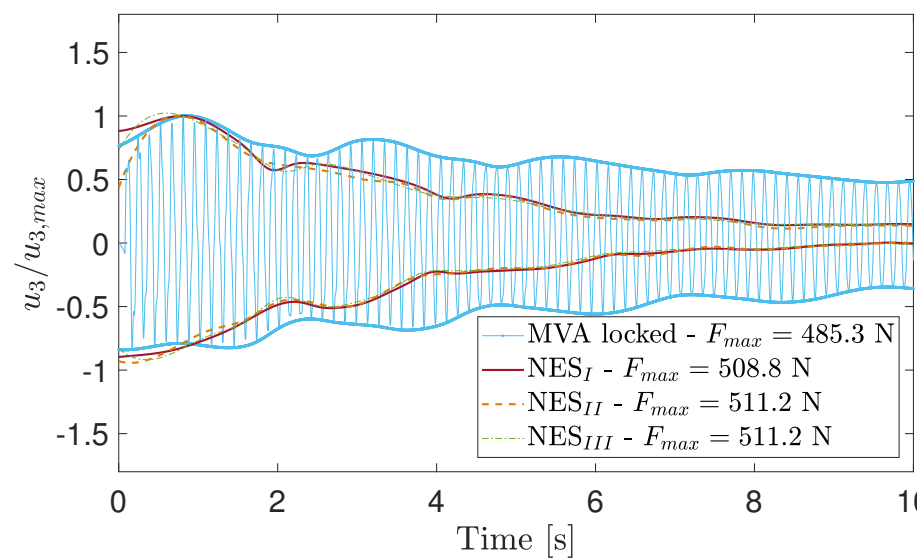

(a)
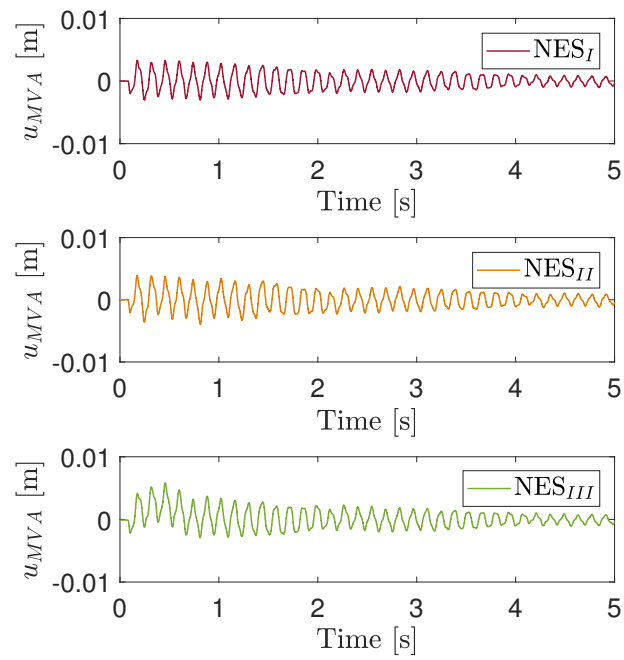

(b)

Figure 8. (a) Normalized displacement of the top floor obtained in the case of shock when the structure is coupled with the different NES configurations, (b) NES displacement.

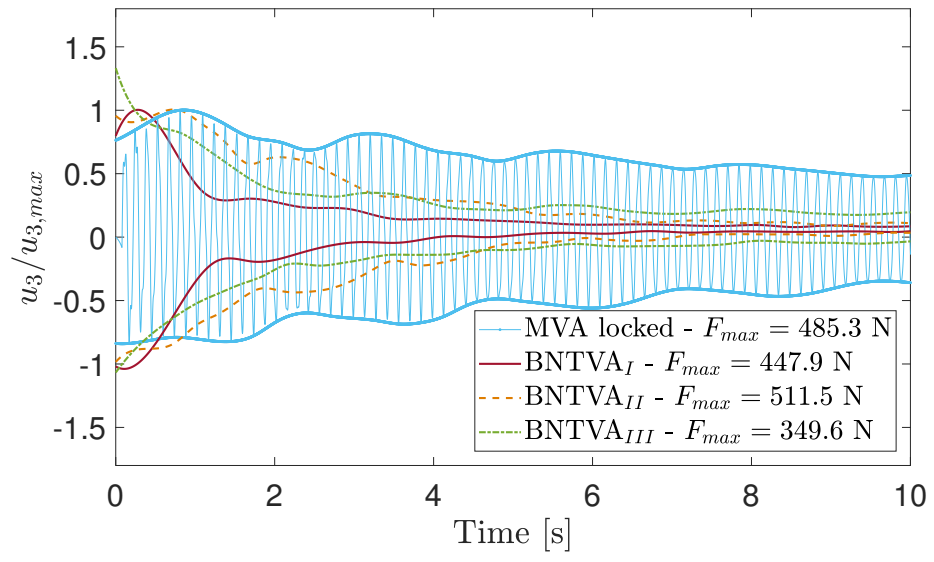

(a)
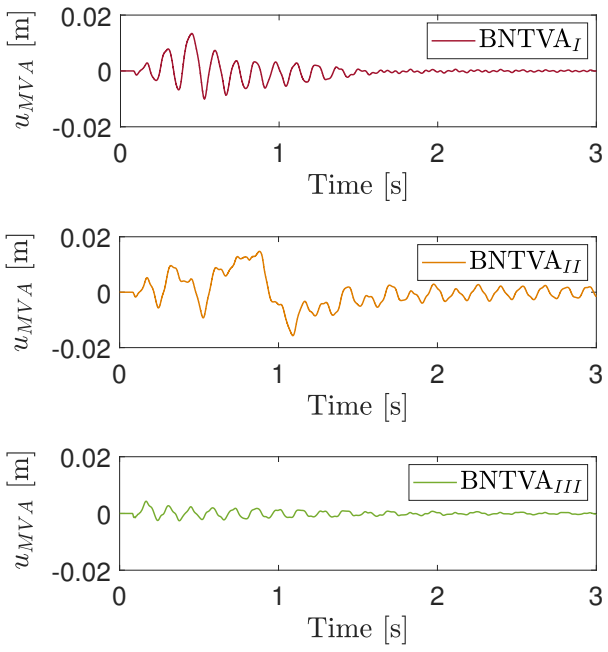

(b)

Figure 9. (a) Normalized displacement of the top floor obtained in the case of shock when the structure is coupled with the different BNTVA configurations, (b) BNTVA displacement from one of the two stable positions.

$$
C_{\bar{f}, f_{1}-f_{3}}=\frac{\sqrt{\int_{\omega_{\min }}^{\omega_{\max }}\left|\frac{u_{3}(\omega)}{F(\omega)}\right|^{2} \mathrm{~d} \omega}}{\sqrt{\int_{\omega_{\min }}^{\omega_{\max }}\left|\frac{u_{0,3}(\omega)}{F_{0}(\omega)}\right|^{2} \mathrm{~d} \omega}} .
$$

where $u_{3}(\omega)$ and $F(\omega)$ are the Fourier transforms of the top floor displacement and of the applied impulse force, respectively, whereas $u_{0,3}(\omega)$ and $F_{0}(\omega)$ denote the same values in the case of locked MVA.

Integrals are evaluated in an angular frequency range bounded by $\omega_{\min }$ and $\omega_{\max }$. These values, reported in Table 4, correspond to a large angular frequency bandwidth for $C_{\bar{f}}$ and to narrow angular frequency band- 
widths around the three modal frequencies in the $y$-direction for $C_{f_{1}}, C_{f_{2}}$, and $C_{f_{3}}$.

For the temporal domain, the performance criteria are given by :

$$
C_{v}=\frac{\int_{\tau_{\min }}^{\tau_{\max }}\left|\frac{\dot{u}_{3}(\tau)}{\dot{u}_{3, \max }}\right|^{2} \mathrm{~d} \tau}{\int_{\tau_{\min }}^{\tau_{\max }}\left|\frac{\dot{u}_{0,3}(\tau)}{\dot{u}_{0,3, \max }}\right|^{2} \mathrm{~d} \tau}, \quad \text { and } \quad C_{d}=\frac{\int_{\tau_{\min }}^{\tau_{\max }}\left|\frac{u_{3}(\tau)}{u_{3, \max }}\right|^{2} \mathrm{~d} \tau}{\int_{\tau_{\min }}^{\tau_{\max }}\left|\frac{u_{0,3}(\tau)}{u_{0,3, \max }}\right|^{2} \mathrm{~d} \tau}
$$

where the top floor velocity $\dot{u}_{3}(\tau)$ and displacement $u_{3}(\tau)$ are normalized by their maximal values $\dot{u}_{3, \max }$ and $u_{3, \max }$, subscripts 0 denoting the same values in the case of locked MVA.

Integrals are evaluated in the $\left[\tau_{\min } \tau_{\max }\right]$ interval where $\tau_{\min }=0 \mathrm{~s}$ and $\tau_{\max }=10 \mathrm{~s}$. It is worth noting that in the case of locked MVA $u_{3}=u_{0,3}$, thus the criteria are equals to 1 . Therefore, the best MVA performances are obtained when the criteria approach 0 .

In order to compare the performance of the different MVA settings reported in Section 2, criteria presented in Eqs. (2)-(3) are applied to the experimental signals and results are shown in Fig. 10. Analysis of the results allows one to draw the following conclusions:

- $C_{\bar{f}}$ and $C_{f_{1}}$ criteria behave in a similar manner, which means that most of the vibrational energy is concentrated around the first mode of vibration;

- all the MVA are very effective for the vibration mitigation of the first resonant frequency, as ascertained by the values attained for $C_{f_{1}}$ criterion. However, the efficacy for the other resonant frequencies is generally poor and case-dependent. In some cases, a slight increase in the vibrational energy can be obtained for secondary peaks (see e.g. the behaviour of $C_{f_{3}}$ for NES and BNTVA);

- the BNTVA configuration is the more sensitive to the selected nonlinear stiffness coefficients;

- vibration reduction attained for all the MVA configurations is also attested in the temporal domain.

From Fig. 10, three configurations were considered globally more efficient and were chosen for a further analysis. The selected settings are NLTVA $I I, \mathrm{NES}_{I I I}$, and $\mathrm{BNTVA}_{I}$, and are denoted hereafter as NLTVA, NES, and BNTVA.

Table 5 and Fig. 11 report the criteria dependency on the force amplitude, for which the performance criteria are evaluated as the ratio of the normalized response of the coupled system to the one with the locked MVA, according to Eqs. (2)-(3). For the considered MVA configurations, the ratios are evaluated for three shock levels, with slight variations of the amplitude. Results show that the MVA is more effective for high loads and, also in this case, around the first mode of vibration. In the temporal domain, the MVA effectiveness clearly increases with the load amplitude when criteria are evaluated in terms of structure displacement. As a result, the NLTVA appears to be the best choice in order to damp structure vibrations around the first mode, whereas the MVA effectiveness decreases for higher modes.

\begin{tabular}{|c|cc|}
\hline & $\omega_{\min } / 2 \pi[H z]$ & $\omega_{\max } / 2 \pi[H z]$ \\
\hline$C_{\bar{f}}$ & 0 & 50 \\
$C_{f_{1}}$ & 6.1 & 8.1 \\
$C_{f_{2}}$ & 19.9 & 21.9 \\
$C_{f_{3}}$ & 30.9 & 32.9 \\
\hline
\end{tabular}

Table 4. Upper and lower integral bounds for the performance criteria in the frequency domain. 


\subsection{Imposed displacement excitation}

In this section, the effect of the MVA to mitigate the primary structure vibrations is investigated in the case of an imposed initial displacement, without initial velocity. This kind of initial conditions significantly increases the vibration amplitudes of the structure, thus a better assessment of the effect of the nonlinearities in the efficacy of the MVA is obtained. Indeed, whereas the maximum displacement of the top floor of the structure attained experimentally in the case of a shock was $3.5 \mathrm{~mm}$, amplitudes up to $7.7 \mathrm{~mm}$ can be reached with the imposed initial conditions.

Figure 12 depicts the signal envelopes of the free vibration displacements of the top floor for the three configurations of the MVA (NLTVA, NES, and BNTVA). The initial condition is imposed by moving the primary structure from its equilibrium position and releasing it, leading to slightly different values of initial accelerations. For this range of initial amplitudes, the three selected tunings of the MVA give rise to little differences in mitigating the primary structure vibrations. However, the signal envelopes clearly show the efficiency of the MVA to rapidly damp the oscillations. One can observe that the NES seems to have the more uniform and efficient decay, whereas oscillations of the signal envelope are noticed during the first instants of the decay for the NLTVA and the BNTVA.

Figure 13 displays the Fourier transforms of the acceleration responses of the top floor for the imposed displacement case. In this case, Hanning window was applied to the experimental signals in order to increase the FFT dynamic range. For the three different tunings of the MVA, the amplitude of the first mode is significantly decreased due to the absorber, showing undoubtedly that the MVA efficiently controls the

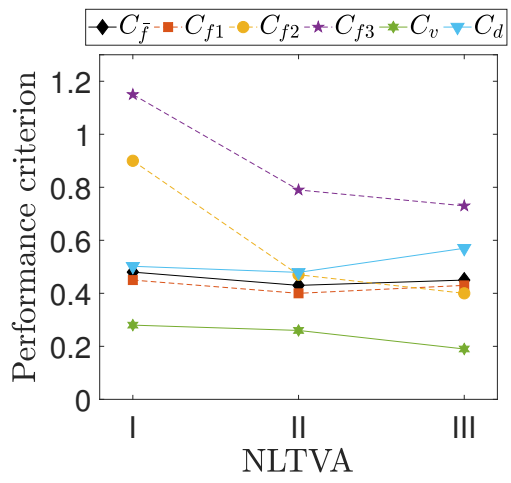

(a)

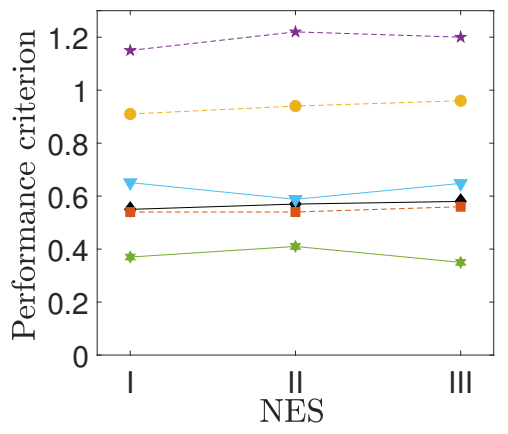

(b)

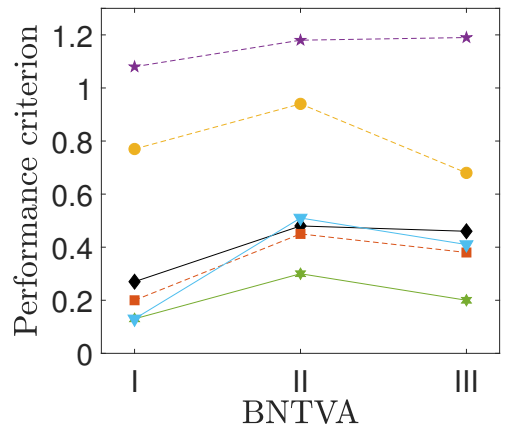

(c)

Figure 10. Performance criteria in the frequency and temporal domains for several MVA configurations (Table 2): (a) NLTVA, (b) NES, and (c) BNTVA. For each MVA setting, linear properties are kept fixed, whereas nonlinear stiffness coefficients are varying.

\begin{tabular}{|c|c|cccccc|}
\hline & $F[N]$ & $C_{\bar{f}}$ & $C_{f_{1}}$ & $C_{f_{2}}$ & $C_{f_{3}}$ & $C_{v}$ & $C_{d}$ \\
\hline \multirow{4}{*}{ NLTVA } & 99 & 0.52 & 0.50 & 0.71 & 1.01 & 0.31 & 0.47 \\
& 316 & 0.45 & 0.38 & 0.73 & 1.00 & 0.28 & 0.34 \\
& 511 & 0.39 & 0.33 & 0.69 & 1.06 & 0.28 & 0.31 \\
\hline \multirow{3}{*}{ NES } & 100 & 0.54 & 0.53 & 0.79 & 1.24 & 0.33 & 0.47 \\
& 283 & 0.44 & 0.42 & 0.71 & 1.13 & 0.30 & 0.39 \\
& 511 & 0.41 & 0.35 & 0.56 & 1.00 & 0.68 & 0.29 \\
\hline \multirow{3}{*}{ BNTVA } & 119 & 0.64 & 0.63 & 0.63 & 0.93 & 0.49 & 0.63 \\
& 296 & 0.46 & 0.42 & 0.76 & 1.04 & 0.24 & 0.35 \\
& 511 & 0.45 & 0.40 & 0.85 & 1.13 & 0.33 & 0.28 \\
\hline
\end{tabular}

Table 5. Values of the performance criteria of the MVA in the frequency and temporal domains for increasing force amplitude. 


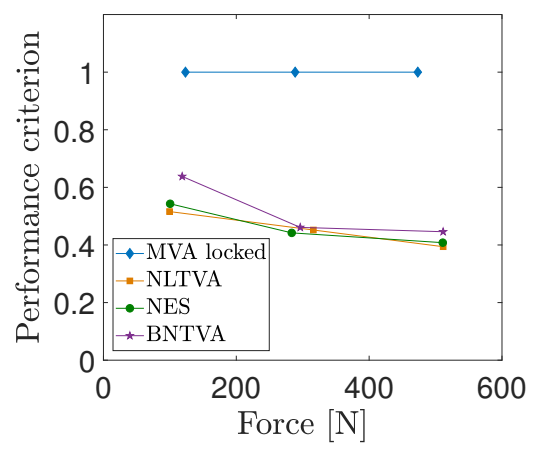

(a)

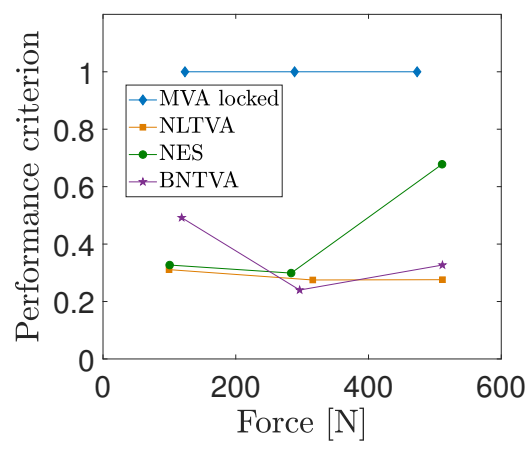

(b)

Figure 11. Performance criteria in the (a) frequency $\left(C_{\bar{f}}\right)$ and (b) temporal $\left(C_{v}\right)$ domains for several MVA configurations (see Table 4).

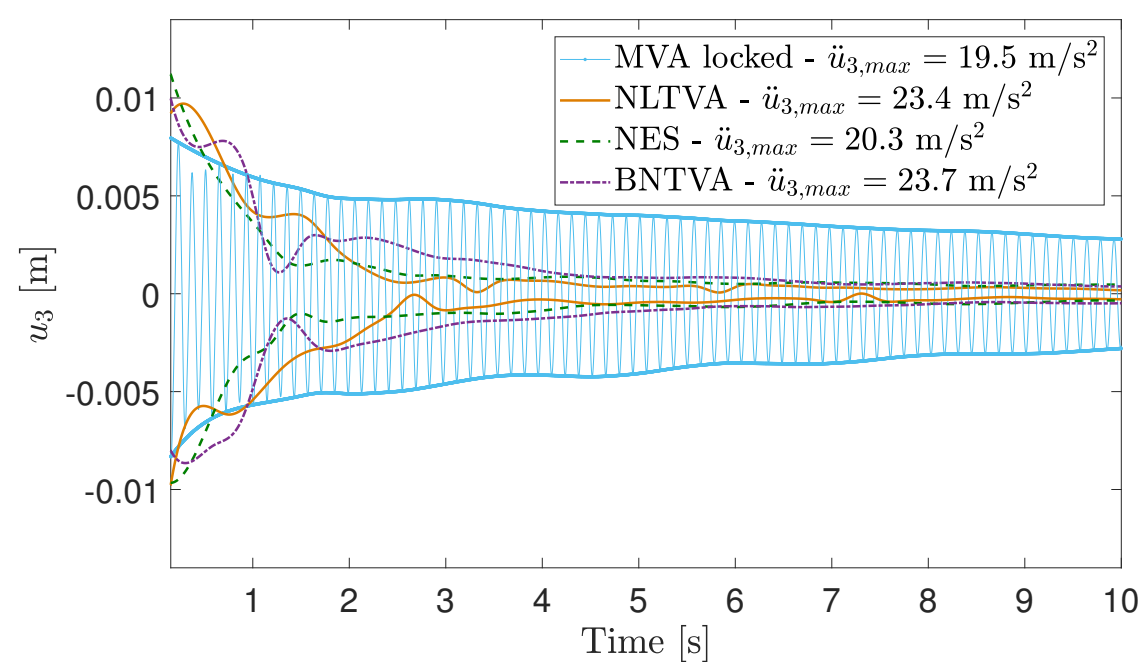

Figure 12. Experimental displacement of the top floor obtained in the case of imposed displacement when the structure is coupled with the MVA for three configurations (NLTVA, NES, and BNTVA) and when the MVA is locked.

first resonance of the structure. More precisely, the resonant peak reduction achieved when the NLTVA is coupled with the structure is of about $11.2 \mathrm{~dB}$, and of about $7.8 \mathrm{~dB}$ and $7 \mathrm{~dB}$ in the cases of NES and BNTVA, respectively. On the contrary, the amplitude of the third mode in the $y$-direction at $31.9 \mathrm{~Hz}$, and of the torsional mode at $14.7 \mathrm{~Hz}$ is increased for each case. The NES having no eigenfrequency, one would have expected a better control of the oscillations for any resonance frequency of the primary structure, but this feature is not observed in our experiments. However, the peak amplitude at $31.9 \mathrm{~Hz}$ increases less importantly than in the case of the NLTVA and the BNTVA. Finally, the BNTVA exhibits a good control also on the second mode in the $y$-direction $(20.9 \mathrm{~Hz})$ and on the second torsional mode $(35.5 \mathrm{~Hz})$, since the peak amplitude is reduced in both cases.

\subsection{Single-frequency excitation}

This section closes the experimental observations by investigating the response of the system to external harmonic forcing, especially single frequency excitation, applied by an electromagnetic shaker linked to the sliding stage on which the structure is installed. Namely, the shaker is connected to a sliding plate carrying the whole structure, and the external excitation is imposed along the $y$-axis, as shown in Fig. 3(a). The behaviour of the MVA at four different excitation frequencies is studied: at $7.1 \mathrm{~Hz}$ and $21 \mathrm{~Hz}$ (first and second 


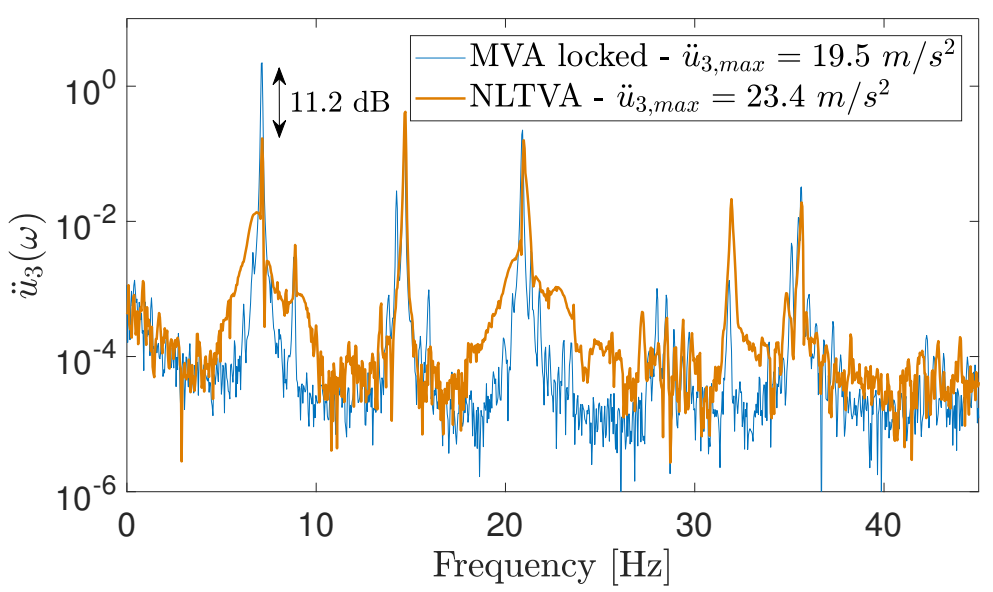

(a)

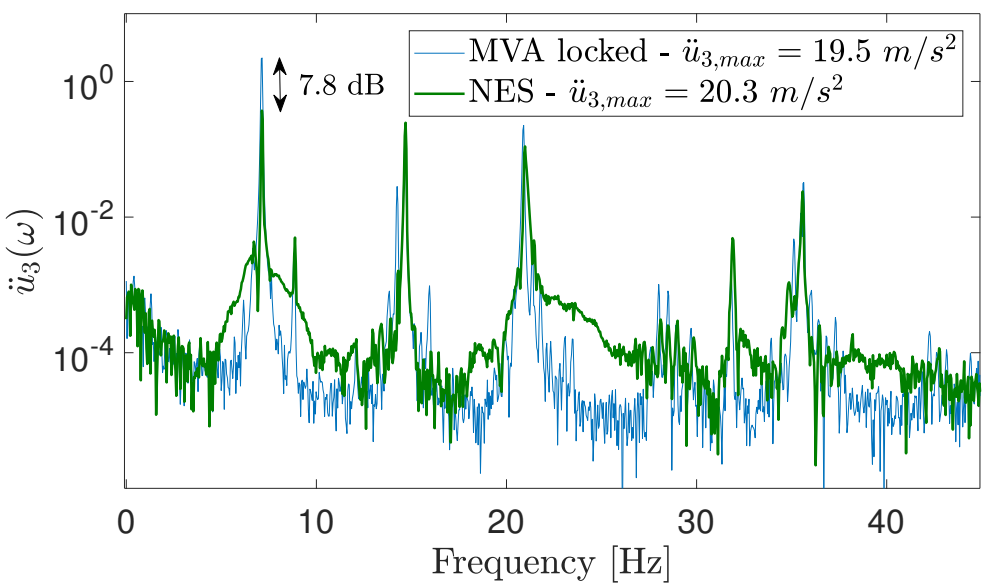

(b)

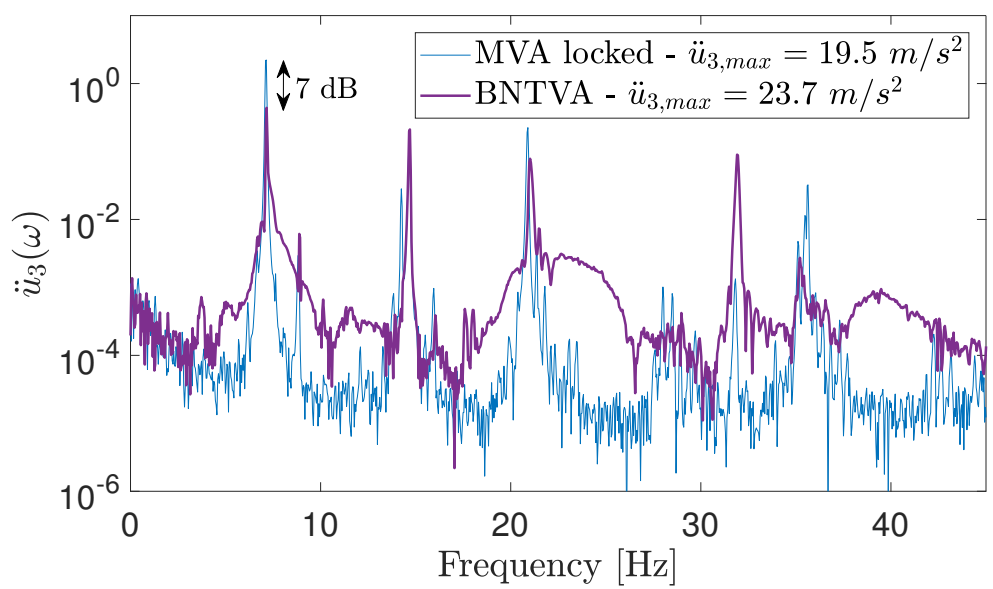

(c)

Figure 13. Fourier transforms of the top floor of the structure in the case of imposed displacement when the structure is coupled with the (a) NLTVA, (b) NES, and (c) BNTVA. 
mode along $y$-axis), $9.1 \mathrm{~Hz}$ (close to the first mode along $x$-axis), and $14.7 \mathrm{~Hz}$ (first torsional mode). The results are reported in Fig. 14, illustrating the multi-storey structure response at the top floor as a function of the ground acceleration imposed by the shaker. Measures are carried out in the permanent regime and for increasing values of the forcing amplitude, thus giving a global view of the mitigation performance of the absorber. Indeed, ground acceleration presented on the horizontal axis denotes the maximum amplitude of the shaker acceleration in stationary regime.

Fig. 14(a) clearly shows the effectiveness of the MVA, whatever its setting, for controlling the vibrations around the fundamental frequency of the primary structure. Indeed, an important decrease of the amplitudes is observed for each case. Furthermore, a mild deviation as compared to the linear behaviour is observed around the second mode along the $y$-axis at $21 \mathrm{~Hz}$ (Fig. 14(b)), the global behaviour being linear. Finally, Figs. 14(c)-14(d) indicate that the MVA has no effect near the first mode along $x$-axis at $9.1 \mathrm{~Hz}$ and on the first torsional mode at $14.7 \mathrm{~Hz}$, which was expected given the direction and the positioning of the MVA at the top floor of the structure. This result was awaited for the NLTVA, which is specifically tuned to control the fundamental eigenfrequency. Moreover, our set-up shows that the NES is not able to damp out vibrations of any frequency. As a matter of fact, we observed during our experiments that the force threshold required to activate the energy transfer is very large at higher frequencies, so that getting over it is very difficult experimentally, as also concluded in [26].

Returning back to Fig. 14(a), one can observe an interesting nonlinear behaviour in the case of the NLTVA. Namely, while increasing the forcing amplitude, a second vibration regime is obtained, with a jump phenomenon switching suddenly from one amplitude to another with improved efficiency. Such behaviour is also present in the case of the BNTVA but to a lesser extent due to the fact that our measurements only show one equilibrium point.

The aim of the next section is to properly investigate this observed behaviour and to explain it with a simple numerical model fitted to the experiments. Consequently, only the case of the NLTVA is considered in the next section.

\subsubsection{Model fitting}

In this section, the behaviour of the NLTVA is obtained by means of numerical simulations performed through a pseudo-arclength continuation method implemented in the AUTO 2000 software [59]. The coupled system is modeled as a two degree of freedom system representing the multi-storey structure and the MVA. The resulting equations of motion are given by:

$$
\left\{\begin{array}{l}
m_{s} \ddot{y}_{s}(t)+c_{s} \dot{y}_{s}(t)+k_{s} y_{s}(t)+c_{a} \dot{z}(t)+\mu_{f r} m_{a} g \operatorname{sign}(\dot{z}(t))+ \\
\quad+k_{a} z(t)+k_{a, 3} z^{3}(t)+k_{a, 5} z^{5}(t)=F \sin (\omega t), \\
m_{a} \ddot{y}_{a}(t)-\mu_{f r} m_{a} g \operatorname{sign}(\dot{z}(t))-c_{a} \dot{z}(t)-k_{a} z(t)-k_{a, 3} z^{3}(t)-k_{a, 5} z^{5}(t)=0 .
\end{array}\right.
$$

where $z(t)=y_{s}(t)-y_{a}(t)$ is the relative displacement between $y_{s}(t)$, the displacement of the structure top floor, and $y_{a}(t)$, that of the MVA; $m_{s}, c_{s}$, and $k_{s}$ are the mass, damping coefficient, and stiffness of the equivalent SDOF oscillator representing the whole structure; $m_{a}$ and $c_{a}$ are the mass and the damping coefficient of the vibration absorber; $\mu_{f r}$ is the sliding friction coefficient; $k_{a}, k_{a, 3}$, and $k_{a, 5}$ are the linear, nonlinear cubic and quintic stiffness coefficients of the MVA; $g$ is the gravitational acceleration, and $F$ and $\omega$ are the amplitude and the angular frequency of the applied force, respectively. The sign symbol stands for the signum function needed to define the friction damping.

Normalizing the equations of motions respectively by the masses $m_{s}$ and $m_{a}$ gives

$$
\left\{\begin{array}{l}
\ddot{y}_{s}(t)+2 \xi_{s} \omega_{s} \dot{y}_{s}(t)+\omega_{s}^{2} y_{s}(t)+ \\
\quad+\mu\left(2 \xi_{a} \omega_{a} \dot{z}(t)+\mu_{f r} g \operatorname{sign}(\dot{z}(t))+\omega_{a}^{2} z(t)+\alpha z^{3}(t)+\beta z^{5}(t)\right)=\bar{F} \sin (\omega t), \\
\ddot{y}_{a}(t)-\mu_{f r} g \operatorname{sign}(\dot{z}(t))-2 \xi_{a} \omega_{a} \dot{z}(t)-\omega_{a}^{2} z(t)-\alpha z^{3}(t)-\beta z^{5}(t)=0 .
\end{array}\right.
$$

where $\mu=m_{a} / m_{s}$ is the mass ratio, $\alpha=k_{a, 3} / m_{a}$ and $\beta=k_{a, 5} / m_{a}$ are the normalized nonlinear stiffness coefficients, $\bar{F}=F / m_{s}$ is the normalized force, and $\omega_{s}=\sqrt{k_{s} / m_{s}}$ and $\omega_{a}=\sqrt{k_{a} / m_{a}}$ are the natural 


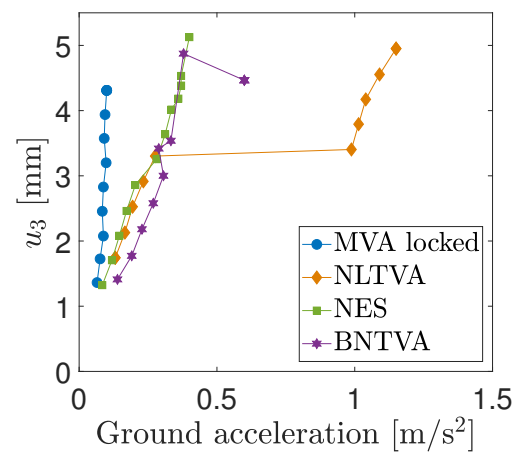

(a)

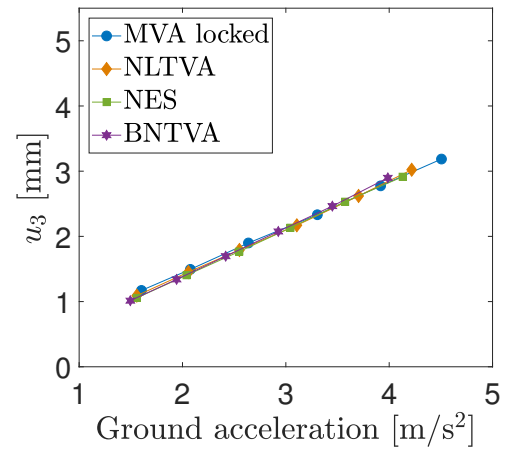

(c)

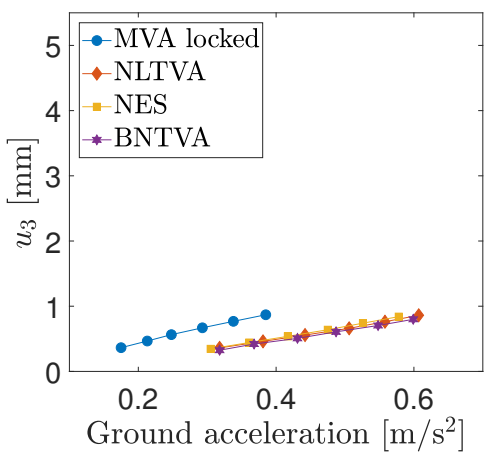

(b)

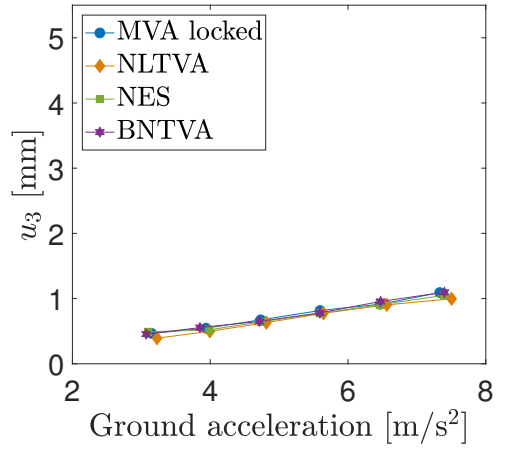

(d)

Figure 14. Experimental displacement response of the top floor in the case of harmonic excitation at (a) $f=7.1 \mathrm{~Hz}$ and (b) $f=21 \mathrm{~Hz}$ (first and second mode along $y$-axis), (c) $f=9.1 \mathrm{~Hz}$ (close to the first mode along $x$-axis), and (d) $f=14.7 \mathrm{~Hz}$ (first torsional mode).

pulsations.

In Fig. 15(a), the experimental displacement of the structure top floor measured by varying the ground acceleration amplitude is compared with numerical results obtained by using parameters indicated in Table 6 . In the identification procedure, the primary structure angular frequency $\omega_{s}$ is kept constant and equal to the frequency of the first mode along the $y$-direction. In addition, the sliding friction coefficient $\mu_{f r}$ was selected below the generic range for static frictional coefficient between lubricated Plexiglas and steel, which is between 0.4 and 0.5. It is worth noting that the mass of the equivalent SDOF oscillator $m_{s}$ considered here corresponds to the modal mass ratio of the first mode. For this reason the mass ratio $\mu$ presented in Table 6 is larger than the value reported in Section 3.1, which was evaluated with respect to the whole structure. All other parameters were chosen so as to improve matching with the experimental curve. With respect to the MVA properties evaluated by means of the multipolar model (see NLTVA $I I I$ in Table 2), an error of $24 \%$ in term of natural frequency was found. This error value is considered acceptable. On the contrary, discrepancy rises for the nonlinear coefficients obtained. This might come from inaccuracy in the multipolar model to be explored, or from the quality of the magnets used in the experiments. Indeed, the local magnetization may be slightly inhomogeneous over the ring so that some minor differences might be awaited from one real magnet to another one.

Fig. 15(a) shows how this simple two degree of freedom model is able to catch the complex nonlinear behaviour of the present structure, where $y_{s}=u_{3}$ according to the numerical model. Indeed, although the shaker power is linearly increased, the ground motion acceleration suddenly rises, jumping to higher amplitudes. The numerical model correctly predicts this jump phenomenon, also showing presence of unstable solutions. Finally, Fig. 15(b) presents a comparison between the numerical and experimental responses 


\begin{tabular}{|cccccccc|}
\hline$\mu[\%]$ & $\xi_{s}[\%]$ & $\omega_{s}[\mathrm{rad} / \mathrm{s}]$ & $\xi_{a}[\%]$ & $\omega_{a}[\mathrm{rad} / \mathrm{s}]$ & $\alpha\left[\mathrm{N} / \mathrm{m}^{3}\right]$ & $\beta\left[\mathrm{N} / \mathrm{m}^{5}\right]$ & $\mu_{f r}$ \\
\hline 2.2 & 0.25 & 44.71 & 0.8 & 33.91 & $4.3 \times 10^{6}$ & $1.1 \times 10^{8}$ & 0.27 \\
\hline
\end{tabular}

Table 6. Properties of the coupled system.

around $7.1 \mathrm{~Hz}$. This analysis was carried out in order to further validate the numerical model and to demonstrate that NLTVA is also effective around the resonant frequency. As a result, it is possible to confirm that vibration absorption is attained for all considered frequencies since, in the case of locked MVA, lower ground accelerations lead to larger displacements of the top floor. Numerical curves also show that adding such a nonlinear absorber greatly modifies the primary mass response to harmonic excitation by giving rise to complex nonlinear phenomena, such as jumps, loops, and instabilities. Thus, the present model is able to predict the overall behaviour of the principal structure for all considered cases.

\section{Conclusions}

In this paper, the effectiveness of a nonlinear Magnetic Vibration Absorber for the passive control of a multi-storey structure is explored experimentally and numerically. The MVA, composed of a moving magnet dipped into a magnetic field induced by others fixed magnets, behaves as a nonlinear SDOF oscillator. Depending on the magnets gap, MVA stiffnesses vary so that the same experimental device can be tuned to the primary structure as NLTVA, NES, and BNTVA. Several MVA configurations were thus placed in turn on the structure top floor and tested under various external excitation, such as shock, imposed displacement, and harmonic force. For the shock tests, the performance of several MVA settings was compared in terms of energy absorption in the frequency and temporal domains. As a result, we can conclude that the MVA is very effective for the vibration control of the first vibration mode of the structure, whereas some amount of energy is transferred to higher modes. The influence of the MVA nonlinearity on the response of the coupled system was also studied. It appears that the NLTVA and the BNTVA nonlinearity affects a lot the overall behaviour of the structure, whereas the NES nonlinearity is not decisive in the free vibration case due to the small amplitude of oscillations. Moreover, the force threshold needed to excite the targeted energy transfer mechanism of the NES has been found to be attained for the first mode, but not for the higher modes. Consequently, the expected broadband passive control of the NES has not been observed experimentally. Then, imposed displacement load conditions had confirmed the MVA effectiveness also for vibrations with larger amplitudes. Finally, the system was harmonically forced at the resonant frequencies. Once again, MVA performed very well around the first mode. In addition, jumps phenomena appeared when the NLTVA was coupled to the structure, really increasing the vibration mitigation effect. The NLTVA behaviour was then explored numerically, by proving its effectiveness also in the proximity of the first mode.

In further studies, a more accurate model for the multi-storey structure should be proposed in order to explore numerically others MVA configurations. Frequency sweeping along a large frequency band may be also performed, as well as transient responses used as excitation, e.g. typical earthquake signals. Concerning the design of the MVA, the next key question to tackle is that of finely tuning the damping of the absorber so as to obtain the optimal passive control characteristics. For example, eddy currents may be used in order to increase the damping of the MVA in a controlable manner. 

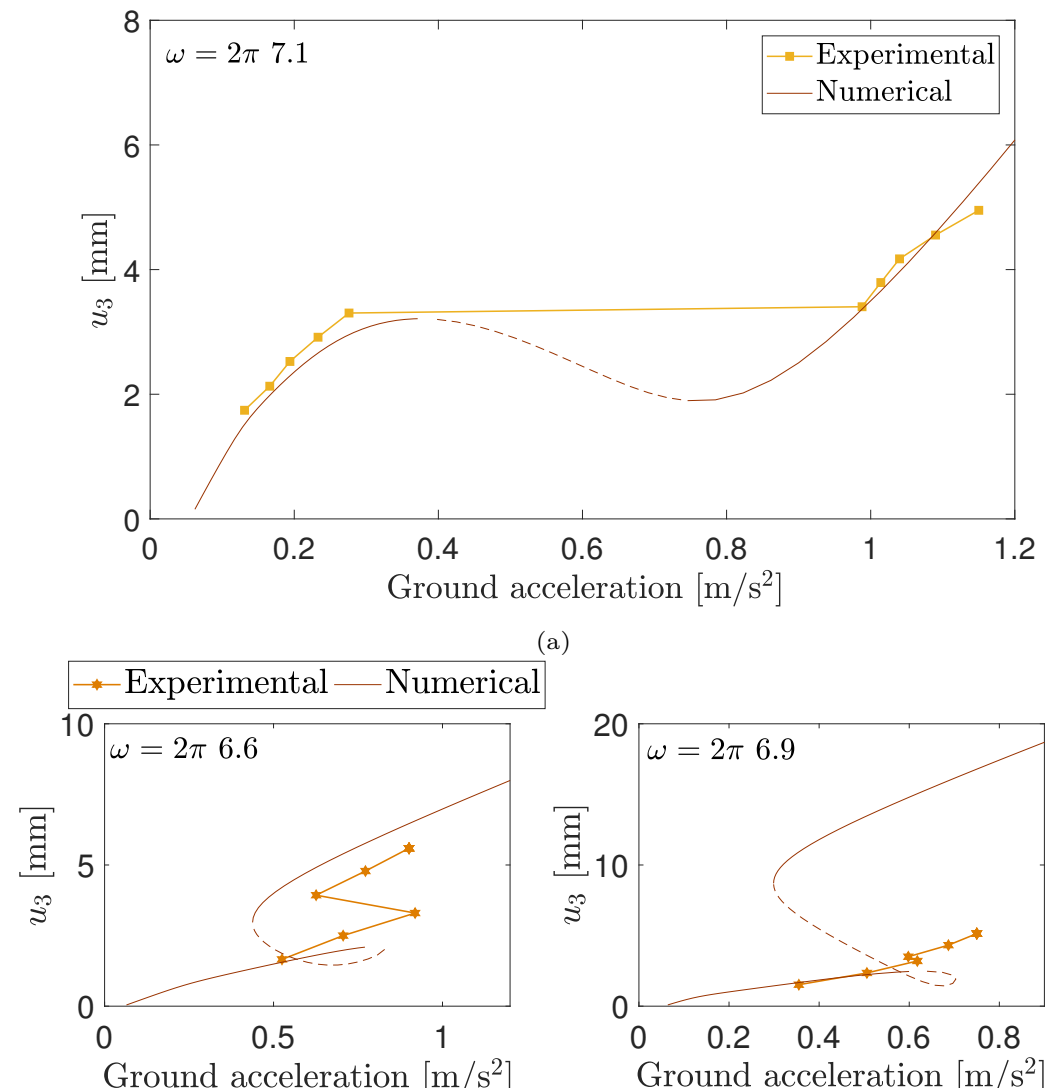

(a)
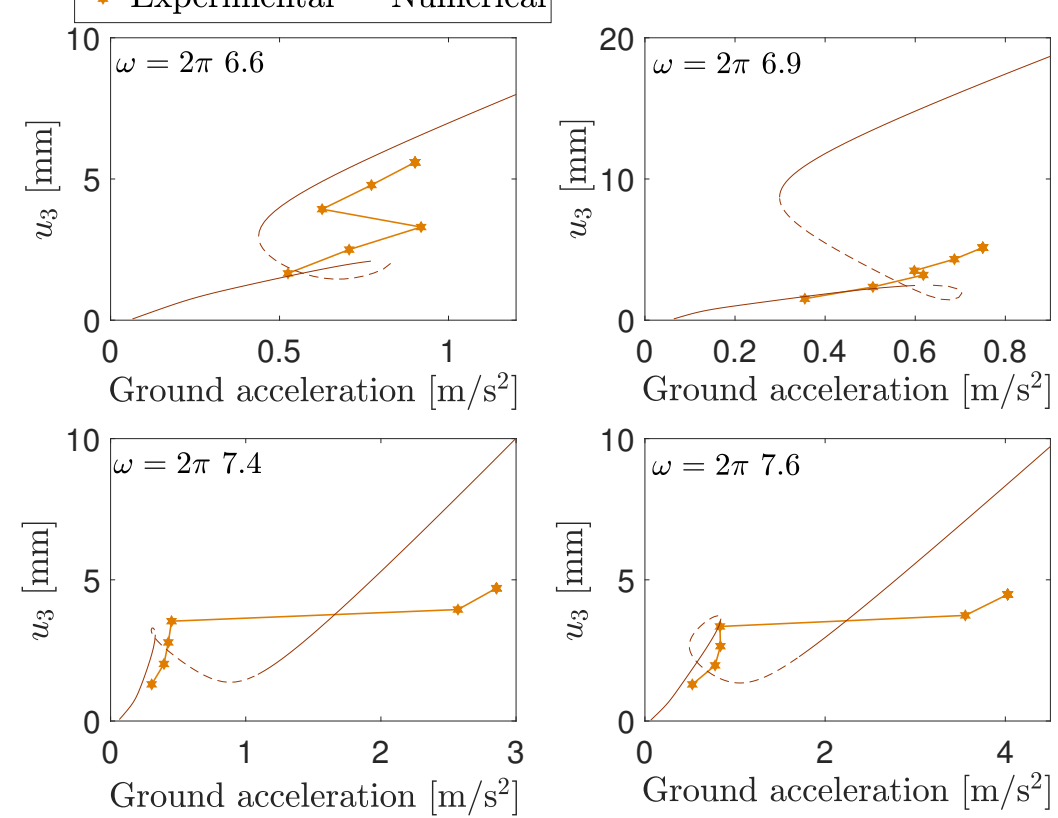

(b)

Figure 15. Multi-storey structure coupled with the NLTVA: experimental and numerical displacement responses of the top floor in the case of harmonic excitation at (a) $f=7.1 \mathrm{~Hz}$ and (b) around the resonance frequency $\left(y_{s}=u_{3}\right)$. 


\section{Appendix}

\section{Appendix A. Multipolar model}

Appendix A.1. Multipolar expansion of cylindrical and ring magnets

The interaction energy $E$ between two similar permanent magnets is given by

$$
E=-\int_{V} \mathbf{M} \cdot \mathbf{B}(\mathbf{p}) \mathrm{d} V
$$

where $V$ is the magnet volume, $\mathbf{p}$ is the vector going from one magnet to the other, $\mathbf{M}$ the magnetization, and $\mathbf{B}$ the magnetic field. By expressing $\mathbf{M}$ as $\mathbf{M}=\mathcal{M} / V$, where $\mathcal{M}$ is the magnetic moment, the force created by the magnetic field $\mathbf{B}$ on the vibrating magnet can be expressed as

$$
\mathbf{F}=\nabla(E)=\nabla(\mathcal{M} \cdot \mathbf{B}(\mathbf{p})) .
$$

When the distance between magnets is larger than their characteristic length, i.e. the radius of the sphere containing the magnet, a multipolar expansion of the magnetic field may be preferred to the dipolar approximation. According to Petruska and Abbot [60], the multipolar expansion of the magnetic field created by a cylindrical magnet may be expressed as:

$$
\begin{aligned}
\mathbf{B}_{\text {dip }}(\mathbf{p})= & \frac{\mu_{0}}{4 \pi} \frac{1}{p^{3}}\left(3 \hat{\boldsymbol{p}} \hat{\boldsymbol{p}}^{T}-\mathbf{I}\right) \mathcal{M}, \\
\mathbf{B}_{\text {quad }}(\mathbf{p})= & \frac{\mu_{0}}{4 \pi} \frac{1}{p^{5}}\left(\frac{L}{2}\right)^{2}\left(\frac{4-3 \beta^{2}}{8}\right)\left\{\left[35\left(\hat{\mathcal{M}}^{T} \hat{\boldsymbol{p}}\right)^{2}-15\right] \hat{\boldsymbol{p}} \hat{\boldsymbol{p}}^{T}-\left[15\left(\hat{\mathcal{M}}^{T} \hat{\boldsymbol{p}}\right)^{2}-3\right] \mathbf{I}\right\} \mathcal{M}, \\
\mathbf{B}_{\text {hex }}(\mathbf{p})= & \frac{\mu_{0}}{4 \pi} \frac{1}{p^{7}}\left(\frac{L}{2}\right)^{4}\left(\frac{15 \beta^{4}-60 \beta^{2}+24}{64}\right)\left\{\left[231\left(\hat{\mathcal{M}}^{T} \hat{\boldsymbol{p}}\right)^{4}-210\left(\hat{\mathcal{M}}^{T} \hat{\boldsymbol{p}}\right)^{2}+35\right] \hat{\boldsymbol{p}} \hat{\boldsymbol{p}}^{T}+\right. \\
& \left.-\left[105\left(\hat{\mathcal{M}}^{T} \hat{\boldsymbol{p}}\right)^{4}-70\left(\hat{\mathcal{M}}^{T} \hat{\boldsymbol{p}}\right)^{2}+5\right] \mathbf{I}\right\} \mathcal{M}, \\
\mathbf{B}_{\text {oct }}(\mathbf{p})= & \frac{\mu_{0}}{4 \pi} \frac{1}{p^{9}}\left(\frac{L}{2}\right)^{6}\left(\frac{64-336 \beta^{2}+280 \beta^{4}-35 \beta^{6}}{1024}\right)\left\{\left[6435\left(\hat{\mathcal{M}}^{T} \hat{\boldsymbol{p}}\right)^{6}-9009\left(\hat{\mathcal{M}}^{T} \hat{\boldsymbol{p}}\right)^{4}\right.\right. \\
& \left.\left.+3465\left(\hat{\mathcal{M}}^{T} \hat{\boldsymbol{p}}\right)^{2}-315\right] \hat{\boldsymbol{p}} \hat{\boldsymbol{p}}^{T}-\left[3003\left(\hat{\mathcal{M}}^{T} \hat{\boldsymbol{p}}\right)^{6}-3465\left(\hat{\mathcal{M}}^{T} \hat{\boldsymbol{p}}\right)^{4}+945\left(\hat{\mathcal{M}}^{T} \hat{\boldsymbol{p}}\right)^{2}-35\right] \mathbf{I}\right\} \mathcal{M} .
\end{aligned}
$$

where $p$ is the distance between the magnets, $\mathbf{I}$ is the identity matrix, the superscript $T$ stands for the transpose, $\beta$ is the ratio of the cylinder diameter to its thickness $(\beta=D / L)$, and $\mu_{0}$ is the void permeability.

By writing the problem in polar coordinates, the local system being centered at the fixed magnet, and after some analytical manipulation, one obtains for the dipolar term :

$$
E_{\text {dip }}(\mathbf{p})=-\frac{\mathcal{M} c_{\text {dip }}}{p^{3}}\left(3 \cos ^{2}(\alpha)-1\right) .
$$

where $c_{d i p}=\mu_{0} \mathcal{M} /(4 \pi)$ and $\alpha$ is the angle between $\mathbf{p}$ and the magnetic moment direction. This is the same result obtained for the spherical magnets.

Similarly, integration of the other terms of the multipolar expansion over the magnet volume leads to the higher order terms. Then, according to Eq. (A.2), the energy gradient leads to the attractive or repulsive force between magnets. For ring magnets, as in the case of the MVA, the magnetic field may be evaluated by computing the magnetic field generated by a cylindrical magnet with diameter $D_{e}$ and positive magnetization $\mathbf{M}$, and by subtracting the magnetic field generated by a cylindrical magnet with diameter $D_{i}$ and negative magnetization $-\mathbf{M}$, representing the hole. 
Appendix A.2. Multipolar model for the MVA

The proposed MVA is composed of eighteen ring magnets and is represented in Fig. 1. In particular, two fixed magnets have their magnetic moment in the same direction of that of the vibrating magnet, which are denoted as "repulsive magnets", whereas twelve magnets, three placed at each corner, have their magnetic moment vector parallel to that of the vibrating magnet. They are denoted as "corrective magnets".

Therefore, the coefficients of the multipolar expansion of the magnetic field generated by the central magnets are:

$$
\begin{aligned}
c_{\text {dip }}^{r, c}= & \frac{\mu_{0}}{4 \pi}\left(\mathcal{M}_{e}^{r, c}-\mathcal{M}_{i}^{r, c}\right), \\
c_{\text {quad }}^{r, c}= & \frac{\mu_{0}}{4 \pi}\left(\frac{L^{r}}{2}\right)^{2}\left[\frac{4\left(\mathcal{M}_{e}^{r, c}-\mathcal{M}_{i}^{r, c}\right)-3\left(\left(\beta_{e}^{r, c}\right)^{2} \mathcal{M}_{e}^{r, c}-\left(\beta_{i}^{r, c}\right)^{2} \mathcal{M}_{i}^{r, c}\right)}{8}\right], \\
c_{h e x}^{r, c}= & \frac{\mu_{0}}{4 \pi}\left(\frac{L^{r}}{2}\right)^{4}\left[\frac{24\left(\mathcal{M}_{e}^{r, c}-\mathcal{M}_{i}^{r, c}\right)+15\left(\left(\beta_{e}^{r, c}\right)^{4} \mathcal{M}_{e}^{r, c}-\left(\beta_{i}^{r, c}\right)^{4} \mathcal{M}_{i}^{r, c}\right)}{64}\right. \\
& \left.-\frac{60\left(\left(\beta_{e}^{r, c}\right)^{2} \mathcal{M}_{e}^{r, c}-\left(\beta_{i}^{r, c}\right)^{2} \mathcal{M}_{i}^{r, c}\right)}{64}\right], \\
c_{o c t}^{r, c}= & \frac{\mu_{0}}{4 \pi}\left(\frac{L^{r}}{2}\right)^{6}\left[\frac{64\left(\mathcal{M}_{e}^{r, c}-\mathcal{M}_{i}^{r, c}\right)-35\left(\left(\beta_{e}^{r, c}\right)^{6} \mathcal{M}_{e}^{r, c}-\left(\beta_{i}^{r, c}\right)^{6} \mathcal{M}_{i}^{r, c}\right)}{1024}\right. \\
& \left.+\frac{280\left(\left(\beta_{e}^{r, c}\right)^{4} \mathcal{M}_{e}^{r, c}-\left(\beta_{i}^{r, c}\right)^{4} \mathcal{M}_{i}^{r, c}\right)-336\left(\left(\beta_{e}^{r, c}\right)^{2} \mathcal{M}_{e}^{r, c}-\left(\beta_{i}^{r, c}\right)^{2} \mathcal{M}_{i}^{r, c}\right)}{1024}\right] .
\end{aligned}
$$

where superscripts $r$ and $c$ stand for repulsive and corrective magnets, respectively, $L^{r, c}$ is the magnet thickness, $\mathcal{M}_{e}^{r, c}$ and $\mathcal{M}_{i}^{r, c}$ are the external and internal magnetic moments, $\beta_{e}^{r, c}=D_{e}^{r, c} / L^{r, c}$ and $\beta_{i}^{r, c}=D_{i}^{r, c} / L^{r, c}$.

In the present study, the magnetic moments of the corrective $\left(\mathcal{M}^{c}\right)$ and repulsive $\left(\mathcal{M}^{r}\right)$ magnets have been evaluated by measuring the magnetic field for the actual ring shape through a fitting procedure carried out with respect to the experimental results, [31]. Therefore, $\mathcal{M}_{e}^{r, c}$ and $\mathcal{M}_{i}^{r, c}$ may be simply obtained from the measured $\mathcal{M}^{r, c}$ as follows:

$$
M^{r, c}=\frac{\mathcal{M}^{r, c}}{\left(V_{e}-V_{i}\right)} \Leftrightarrow \mathcal{M}_{e, i}^{r, c}=M^{r, c} V_{e, i}
$$

where $V_{e}$ and $V_{i}$ are the volume of the external and the internal cylinders.

In our case, three similar magnets are joined together at each corner of the MVA in order to increase the corrective effect. Thus, an equivalent magnet of thickness $3 L$ may be considered. Consequently, according to Fig. 1(a), the moving magnet 1 undergoes the following total force:

$$
\mathbf{F}_{T o t}=\sum_{i=2}^{7} \mathbf{F}\left(\mathbf{p}_{\mathbf{i}}\right) \text {. }
$$

where the distances $p_{i}$ can be expressed as functions of the geometrical parameters defining the geometry of the MVA only, i.e. $p_{r}, p_{c}$, and $d$.

Geometrical relationships easily provide:

$$
\begin{aligned}
p_{2} & =p_{r}+\bar{y}, \quad p_{3}=p_{r}-\bar{y} \\
p_{4,6} & =\sqrt{\left(p_{c}+\bar{y}\right)^{2}+d^{2}}, \quad p_{5,7}=\sqrt{\left(p_{c}-\bar{y}\right)^{2}+d^{2}}, \\
p_{c} & =p_{r}-L .
\end{aligned}
$$


where the $\bar{y}$-axis owns to the coordinate system centered on the moving magnet, as shown in Fig. 1(a), and $L$ is the thickness of one magnet.

Note that the versatility of the system relies on the fact that a different number of isolated magnets can be used. This allows for example to increase the mass of the moving magnet (as seen in Fig. 1(b) where a chain of four magnets have been used for the moving magnet), or to increase the magnetic force of the corrective magnets.

In order to obtain the total force $\mathbf{F}_{\text {Tot }}$ up to the quintic term, a Taylor series expansion is used around the rest position $\bar{y}=0$. Finally, the following expression is obtained for the longitudinal force along the $\bar{y}$-axis :

$$
\begin{aligned}
F_{T o t} & \approx-\left(K_{1}^{r}\left(p_{r}\right)-K_{1}^{c}\left(p_{c}, d\right)\right) \bar{y}-\left(K_{3}^{r}\left(p_{r}\right)-K_{3}^{c}\left(p_{c}, d\right)\right) \bar{y}^{3}-\left(K_{5}^{r}\left(p_{r}\right)-K_{5}^{c}\left(p_{c}, d\right)\right) \bar{y}^{5} \\
& \approx-K_{1}\left(p_{r}, p_{c}, d\right) \bar{y}-K_{3}\left(p_{r}, p_{c}, d\right) \bar{y}^{3}-K_{5}\left(p_{r}, p_{c}, d\right) \bar{y}^{5} .
\end{aligned}
$$

The system being symmetric with respect to the rest position, only odd coefficients appear in the restoring force. The linear stiffness coefficient, $K_{1}$, and the nonlinear cubic and quintic stiffness coefficients, $K_{3}$ and $K_{5}$, can be expressed as functions of the distances $d$ and $p_{r}$ only [31], being $p_{c}$ related to $p_{r}$. In particular:

$$
\begin{aligned}
& K_{1}^{r}=K_{1, \text { dip }}^{r}+K_{1, \text { quad }}^{r}+K_{1, \text { hex }}^{r}+K_{1, \text { oct }}^{r}, \\
& K_{1}^{c}=K_{1, \text { dip }}^{c}+K_{1, \text { quad }}^{c}+K_{1, \text { hex }}^{c}+K_{1, \text { oct }}^{c}, \\
& K_{3}^{r}=K_{3, \text { dip }}^{r}+K_{3, \text { quad }}^{r}+K_{3, \text { hex }}^{r}+K_{3, \text { oct }}^{p}, \\
& K_{3}^{c}=K_{3, \text { dip }}^{c}+K_{3, \text { quad }}^{c}+K_{3, \text { hex }}^{c}+K_{3, \text { oct }}^{c}, \\
& K_{5}^{r}=K_{5, \text { dip }}^{r}+K_{5, \text { quad }}^{r}+K_{5, \text { hex }}^{r}+K_{5, \text { oct }}^{r}, \\
& K_{5}^{c}=K_{5, \text { dip }}^{c}+K_{5, \text { quad }}^{c}+K_{5, \text { hex }}^{c}+K_{5, \text { oct }}^{c} .
\end{aligned}
$$

where

$$
\begin{aligned}
& K_{1, \text { dip }}^{r}=48 c_{\text {dip }}^{r} \mathcal{M}^{r} \frac{1}{p_{r}^{5}}, \quad K_{3, \text { dip }}^{r}=240 c_{\text {dip }}^{r} \mathcal{M}^{r} \frac{1}{p_{r}^{7}}, \quad K_{5, \text { dip }}^{r}=672 c_{d i p}^{r} \mathcal{M}^{r} \frac{1}{p_{r}^{9}}, \\
& K_{1, \text { quad }}^{r}=480 c_{\text {quad }}^{r} \mathcal{M}^{r} \frac{1}{p_{r}^{7}}, \quad K_{3, \text { quad }}^{r}=4480 c_{\text {quad }}^{r} \mathcal{M}^{r} \frac{1}{p_{r}^{9}}, \quad K_{5, \text { quad }}^{r}=20160 c_{\text {quad }}^{r} \mathcal{M}^{r} \frac{1}{p_{r}^{11}}, \\
& K_{1, \text { hex }}^{r}=1792 c_{\text {hex }}^{r} \mathcal{M}^{r} \frac{1}{p_{r}^{9}}, \quad K_{3, h e x}^{r}=26880 c_{\text {hex }}^{r} \mathcal{M}^{r} \frac{1}{p_{r}^{11}}, \quad K_{5, h e x}^{r}=177408 c_{h e x}^{r} \mathcal{M}^{r} \frac{1}{p_{r}^{13}}, \\
& K_{1, \text { oct }}^{r}=136440 c_{\text {oct }}^{r} \mathcal{M}^{r} \frac{1}{p_{r}^{11}}, \quad K_{3, \text { oct }}^{r}=3001680 c_{\text {oct }}^{r} \mathcal{M}^{r} \frac{1}{p_{r}^{13}}, \quad K_{5, \text { oct }}^{r}=27315288 c_{\text {oct }}^{r} \mathcal{M}^{r} \frac{1}{p_{r}^{15}} .
\end{aligned}
$$

and 


$$
\begin{aligned}
& K_{1, d i p}^{c}=12 c_{d i p}^{c} \mathcal{M}^{c}\left[\frac{8 p_{c}^{4}-24 d^{2} p_{c}^{2}+3 d^{4}}{\left(p_{c}^{2}+d^{2}\right)^{9 / 2}}\right] \text {, } \\
& K_{3, d i p}^{c}=30 c_{d i p}^{c} \mathcal{M}^{c}\left[\frac{16 p_{c}^{6}-120 d^{2} p_{c}^{4}+90 d^{4} p_{c}^{2}-5 d^{6}}{\left(p_{c}^{2}+d^{2}\right)^{13 / 2}}\right] \text {, } \\
& K_{5, d i p}^{c}=\frac{21}{2} c_{d i p}^{c} \mathcal{M}^{c}\left[\frac{128 p_{c}^{8}-1792 d^{2} p_{c}^{6}+3360 d^{4} p_{c}^{4}-1120 d^{6} p_{c}^{2}+35 d^{8}}{\left(p_{c}^{2}+d^{2}\right)^{17 / 2}}\right] \text {, } \\
& K_{1, q u a d}^{c}=60 c_{\text {quad }}^{c} \mathcal{M}^{c}\left[\frac{16 p_{c}^{6}-120 p_{c}^{4} d^{2}+90 p_{c}^{2} d^{4}-5 d^{6}}{\left(p_{c}^{2}+d^{2}\right)^{13 / 2}}\right] \text {, } \\
& K_{3, \text { quad }}^{c}=70 c_{\text {quad }}^{c} \mathcal{M}^{c}\left[\frac{128 p_{c}^{8}-1792 p_{c}^{6} d^{2}+3360 p_{c}^{4} d^{4}-1120 p_{c}^{2} d^{6}+35 d^{8}}{\left(p_{c}^{2}+d^{2}\right)^{17 / 2}}\right], \\
& K_{5, \text { quad }}^{c}=\frac{315}{2} c_{\text {quad }}^{c} \mathcal{M}^{c}\left[\frac{256 p_{c}^{10}-5760 p_{c}^{8} d^{2}+20160 p_{c}^{6} d^{4}-16800 p_{c}^{4} d^{6}+3150 p_{c}^{2} d^{8}-63 d^{10}}{\left(p_{c}^{2}+d^{2}\right)^{21 / 2}}\right] \text {, } \\
& K_{1, h e x}^{c}=28 c_{\text {hex }}^{c} \mathcal{M}^{c}\left[\frac{128 p_{c}^{8}-1792 p_{c}^{6} d^{2}+3360 p_{c}^{4} d^{4}-1120 p_{c}^{2} d^{6}+35 d^{8}}{\left(p_{c}^{2}+d^{2}\right)^{17 / 2}}\right] \text {, } \\
& K_{3, \text { hex }}^{c}=210 c_{\text {hex }}^{c} \mathcal{M}^{c}\left[\frac{256 p_{c}^{10}-5760 p_{c}^{8} d^{2}+20160 p_{c}^{6} d^{4}-16800 p_{c}^{4} d^{6}+3150 p_{c}^{2} d^{8}-63 d^{10}}{\left(p_{c}^{2}+d^{2}\right)^{21 / 2}}\right] \text {, } \\
& K_{5, \text { hex }}^{c}=\frac{693}{2} c_{\text {hex }}^{c} \mathcal{M}^{c}\left[\frac{1024 p_{c}^{12}-33792 p_{c}^{10} d^{2}+190080 p_{c}^{8} d^{4}-295680 p_{c}^{6} d^{6}+138600 p_{c}^{4} d^{8}}{\left(p_{c}^{2}+d^{2}\right)^{25 / 2}}\right. \\
& \left.-\frac{16632 p_{c}^{2} d^{10}-231 d^{12}}{\left(p_{c}^{2}+d^{2}\right)^{25 / 2}}\right] \text {. } \\
& K_{1, o c t}^{c}=180 c_{o c t}^{c} \mathcal{M}^{c}\left[\frac{1516 p_{c}^{10}-2694 p_{c}^{8} d^{2}+21826 p_{c}^{6} d^{4}-17598 p_{c}^{4} d^{6}+2520 p_{c}^{2} d^{8}-35 d^{10}}{\left(p_{c}^{2}+d^{2}\right)^{21 / 2}}\right], \\
& K_{3, o c t}^{c}=990 c_{o c t}^{c} \mathcal{M}^{c}\left[\frac{6064 p_{c}^{12}-26848 p_{c}^{10} d^{2}+182870 p_{c}^{8} d^{4}-308630 p_{c}^{6} d^{6}+136850 p_{c}^{4} d^{8}}{\left(p_{c}^{2}+d^{2}\right)^{25 / 2}}\right. \\
& \left.-\frac{14602 p_{c}^{2} d^{10}-175 d^{12}}{\left(p_{c}^{2}+d^{2}\right)^{25 / 2}}\right] \text {. } \\
& K_{5, \text { oct }}^{c}=\frac{9009}{2} c_{\text {oct }}^{c} \mathcal{M}^{c}\left[\frac{12128 p_{c}^{14}-94864 p_{c}^{12} d^{2}+725508 p_{c}^{10} d^{4}-1944330 p_{c}^{8} d^{6}+1706070 p_{c}^{6} d^{8}}{\left(p_{c}^{2}+d^{2}\right)^{29 / 2}}\right. \\
& \left.-\frac{492594 p_{c}^{4} d^{10}-38712 p_{c}^{2} d^{12}+369 d^{14}}{\left(p_{c}^{2}+d^{2}\right)^{29 / 2}}\right] .
\end{aligned}
$$

It is worth noting that the fourth-order term of the multipolar expansion has been corrected with respect to the value presented in [31].

\section{References}

[1] J. Den Hartog, Mechanical Vibrations, McGraw - Hill, New-York, 1934.

[2] H. Frahm, Device for damping vibrations of bodies, Tech. rep., US No. Patent 989958 (1909).

[3] J. Ormondroyd, J. Den Hartog, The theory of the dynamic vibration absorber, Transactions of ASME 50 (1928) 9 - 22. 
[4] T. Asami, O. Nishihara, A. Baz, Analytical solutions to $\mathrm{H} \infty$ and $\mathrm{H}_{2}$ optimization of dynamic vibration absorbers attached to damped linear systems, J. Vib. Acoust 124 (2) (2002) $284-295$.

[5] M. Zilletti, S. Elliott, E. Rustighi, Optimisation of dynamic vibration absorbers to minimise kinetic energy and maximise internal power dissipation, Journal of Sound and Vibration 331 (18) (2012) 4093 - 4100.

[6] Y. Fujino, M. Abe, Design formulas for tuned mass dampers based on a perturbation technique, Earthquake Eng Struct Dyn $22(10)(1993) 833-854$.

[7] S. Krenk, Frequency analysis of the tuned mass damper, Journal of Applied Mechanics 72 (6) (2005) $936-942$.

[8] M. Hadi, Y. Arfiadi, Optimum design of absorber for MDOF structures, Journal of Structural Engineering 124 (11) (1998) $1272-1280$.

[9] R. Rana, T. Soong, Parametric study and simplified design of tuned mass dampers, Engineering Structures 20 (3) (1998) $193-204$

[10] F. Sadek, B. Mohraz, A. Taylor, R. Chung, A method of estimating the parameters of tuned mass dampers for seismic applications, Earthq Eng Struct Dyn 26 (6) (1997) 617 - 636.

[11] C. Lee, Y. Chen, L. Chung, Y. Wang, Optimal design theories and applications of tuned mass dampers, Engineering Structures 28 (1) (2006) $43-53$.

[12] G. Bekdaş, S. Nigdeli, Estimating optimum parameters of tuned mass dampers using harmony search, Engineering Structures 33 (9) (2011) $2716-2723$.

[13] B. Ebrahimi, M. Khamesee, M. Golnaraghi, Design and modeling of a magnetic shock absorber based on eddy current damping effect, Journal of Sound and Vibration 315 (4 - 5) (2008) $875-889$.

[14] J. Bae, J. Hwang, et al., Vibration suppression of a cantilever beam using magnetically tuned-mass-damper, Journal of Sound and Vibration 331 (26) (2012) 5669 - 5684.

[15] Z. Wang, Z. Chen, J. Wang, Feasibility study of a large-scale tuned mass damper with eddy current damping mechanism, Earthq Eng Eng Vib 11 (3) (2012) $391-401$.

[16] X. Lu, B. Huang, Q. Zhang, X. Lu, Experimental and analytical study on vibration control effects of eddy-current tuned mass dampers under seismic excitations, Journal of Sound and Vibration 421 (2018) 153 - 165.

[17] S. Lo Feudo, A. Allani, G. Cumunel, et al., Experimental analysis of a tuned mass damper with eddy currents damping effect, in: Models, Simulation, and Experimental Issues in Structural Mechanics, Vol. 8 of Springer Series in Solid and Structural Mechanics, Springer, Cham, pp. 235 - 248.

[18] S. Krenk, J. Hogsberg, Tuned mass absorber on a flexible structure, Journal of Sound and Vibration 333 (2014) 1577 1595.

[19] F. Bourquin, G. Caruso, M. Peigney, D. Siegert, Magnetically tuned mass dampers for optimal vibration damping of large structures, Smart Materials and Structures 23 (8) (2014) 085009.

[20] H. Yu, F. Gillot, M. Ichchou, Reliability based robust design optimization for tuned mass damper in passive vibration control of deterministic/uncertain structures, Journal of Sound and Vibration 332 (9) (2013) 2222 - 2238.

[21] A. F. Vakakis, L. I. Manevitch, O. Gendelman, L. Bergman, Dynamics of linear discrete systems connected to local, essentially non-linear attachments, Journal of Sound and Vibration 264 (2003) $559-577$.

[22] O. Gendelman, L. I. Manevitch, A. F. Vakakis, R. M. Closkey, Energy pumping in nonlinear mechanical oscillators, I: dynamics of the underlying Hamiltonian systems, Journal of Applied Mechanics 68 (1) (2001) $34-41$.

[23] A. F. Vakakis, O. V. Gendelman, L. A. Bergman, D. M. McFarland, G. Kerschen, Y. S. Lee, Nonlinear targeted energy transfer in mechanical and structural systems, Springer, New - York, 2008.

[24] A. F. Vakakis, O. Gendelman, Energy pumping in nonlinear mechanical oscillators, II: resonance capture, Journal of Applied Mechanics 68 (1) (2001) $42-48$.

[25] E. Gourdon, N. A. Alexander, C. A. Taylor, C. H. Lamarque, S. Pernot, Nonlinear energy pumping under transient forcing with strongly nonlinear coupling: Theoretical and experimental results, Journal of Sound and Vibration $300(3-5)(2007)$ $522-551$.

[26] P. O. Mattei, R. Ponçot, M. Pachebat, R. Côte, Nonlinear targeted energy transfer of two coupled cantilever beams coupled to a bistable light attachment, Journal of Sound and Vibration 373 (2016) $29-51$.

[27] L. I. Manevitch, G. Sigalov, F. Romeo, L. A. Bergman, A. F. Vakakis, Dynamics of a linear oscillator coupled to a bistable light attachment: analytical study, Journal of Applied Mechanics 81 (4) (2013) 041011.

[28] F. Romeo, G. Sigalov, L. A. Bergman, A. F. Vakakis, Dynamics of a linear oscillator coupled to a bistable light attachment: Numerical study, Journal of Computational and Nonlinear Dynamics 10 (1) (2014) 011007.

[29] G. Habib, F. Romeo, The tuned bistable nonlinear energy sink, Nonlinear Dynamics 89 (1) (2017) $179-196$.

[30] F. Romeo, L. I. Manevitch, L. A. Bergman, A. Vakakis, Transient and chaotic low-energy transfers in a system with bistable nonlinearity, Chaos: An Interdisciplinary Journal of Nonlinear Science 25 (5) (2015) 053109.

[31] S. Benacchio, A. Malher, J. Boisson, C. Touzé, Design of a Magnetic Vibration Absorber with tunable stiffnesses, Nonlinear Dynamics 85 (2016) $893-911$.

[32] R. L. Harne, K. W. Wang, A review of the recent research on vibration energy harvesting via bistable systems, Smart Materials and Structures 22 (2013) 023001.

[33] H. J. Rice, J. R. McCraith, Practical non-linear vibration absorber design, Journal of Sound and Vibration 116 (3) (1987) $545-559$.

[34] I. N. Jordanov, B. I. Cheshankov, Optimal design of linear and non-linear dynamic vibration absorbers, Journal of Sound and Vibration 123 (1) (1988) $157-170$.

[35] J. Shaw, S. W. Shaw, A. G. Haddow, On the response of the non-linear vibration absorber, International Journal of Non-Linear Mechanics 24 (4) (1989) $281-293$.

[36] S. Natsiavas, Steady state oscillations and stability of non-linear dynamic vibration absorbers, Journal of Sound and 
Vibration 156 (2) (1992) $227-245$.

[37] N. A. Alexander, F. Schilder, Exploring the performance of a nonlinear tuned mass damper, Journal of Sound and Vibration $319(1$ - 2) (2009) $445-462$.

[38] G. Gatti, M. J. Brennan, I. Kovacic, On the interaction of the responses at the resonance frequencies of a nonlinear two degrees-of-freedom system, Physica D: Nonlinear Phenomena 239 (10) (2010) 591 - 599.

[39] G. Habib, T. Detroux, R. Viguié, G. Kerschen, Nonlinear generalization of Den Hartog's equal-peak method, Mechanical systems and signal processing $52-53$ (2015) $17-28$.

[40] R. Viguié, G. Kerschen, Nonlinear vibration absorber coupled to a nonlinear primary system: A tuning methodology, Journal of Sound and Vibration 326 (3 - 5) (2009) 780 - 793.

[41] G. Habib, G. Kerschen, Suppression of limit cycle oscillations using the nonlinear tuned vibration absorber, Proceedings of the Royal Society of London A 471 (2176).

[42] G. Habib, G. Kerschen, A principle of similarity for nonlinear vibration absorbers, Physica D: Nonlinear Phenomena 332 (2016) $1-8$.

[43] T. Detroux, G. Habib, L. Masset, G. Kerschen, Performance, robustness and sensitivity analysis of the nonlinear tuned vibration absorber, Mechanical Systems and Signal Processing 60 - 61 (2015) $799-809$.

[44] A. Malher, C. Touzé, O. Doaré, G. Habib, G. Kerschen, Flutter control of a two-degrees-of-freedom airfoil using a nonlinear tuned vibration absorber, ASME Journal of Computational and Nonlinear Dynamics 12 (5) (2017) 051016 $051016-11$.

[45] D. M. McFarland, L. A. Bergman, A. F. Vakakis, Experimental study of non-linear energy pumping occurring at a single fast frequency, Int. Journal of Non-linear Mechanics 40 (2005) $891-899$.

[46] Y. Lee, F. Nucera, A. Vakakis, D. McFarland, L. Bergman, Periodic orbits, damped transitions and targeted energy transfers in oscillators with vibro-impact attachments, Physica D: Nonlinear Phenomena 238 (18) (2009) 1868 - 1896

[47] C. Lamarque, O. Gendelman, A. Ture Savadkoohi, E. Etcheverria, Targeted energy transfer in mechanical systems by means of non-smooth nonlinear energy sink, Acta Mechanica $221(1-2)(2011) 175-200$.

[48] E. Gourc, G. Michon, S. Séguy, A. Berlioz, Targeted energy transfer under harmonic forcing with a vibro-impact nonlinear energy sink: Analytical and experimental developments, Journal of Vibration and Acoustics 137 (3) (2015) 031008.

[49] M. A. Al-Shudeifat, A. F. Vakakis, L. A. Bergman, Shock mitigation by means of low- to high-frequency nonlinear targeted energy transfers in a large-scale structure, Journal of Computational and Nonlinear Dynamics 11 (2) (2015) 021006.

[50] G. Pennisi, C. Stephan, E. Gourc, G. Michon, Experimental investigation and analytical description of a vibro-impact NES coupled to a single-degree-of-freedom linear oscillator harmonically forced, Nonlinear Dynamics 88 (3) (2017) 1769 -1784 .

[51] G. Pennisi, B. P. Mann, N. Naclerio, C. Stephan, G. Michon, Magnetic-strung NES with energy harvesting: theoretical and experimental study of a new concept of nonlinear vibration absorber, Journal of Sound and Vibration.

[52] D. Qiu, S. Séguy, M. Paredes, Tuned nonlinear energy sink with conical spring: Design theory and sensitivity analysis, Journal of Mechanical Design 140 (1) (2018) 011404.

[53] C. Grappasonni, G. Habib, T. Detroux, G. Kerschen, Experimental demonstration of a 3D-printed nonlinear tuned vibration absorber, in: Proceedings of the International Modal Analysis Conference (IMAC) XXXIII, Orlando, 2015.

[54] P. Soltani, G. Kerschen, The nonlinear piezoelectric tuned vibration absorber, Smart Materials and Structures 24 (7) (2015) 075015.

[55] H. Jo, H. Yabuno, Amplitude reduction of primary resonance of nonlinear oscillator by a dynamic vibration absorber using nonlinear coupling, Nonlinear Dynamics 55 (1 - 2) (2009) 67 - 78.

[56] M. A. Al-Shudeifat, Asymmetric magnet-based nonlinear energy sink, Journal of Computational and Nonlinear Dynamics 10 (1) (2014) 014502.

[57] I. Yamakawa, S. Takeda, H. Kojima, Behaviour of a new type dynamic vibration absorber consisting of three permanent magnets, Bulletin of JSME 20 (146) (1977) 947 - 954.

[58] T. P. Le, P. Argoul, Continuous wavelet transform for modal identification using free decay response, Journal of Sound and Vibration $277(1-2)(2004) 73-100$.

[59] E. J. Doedel, R. C. Paffenroth, et al., AUTO 2000: Continuation and bifurcation software for ordinary differential equations (with HomCont), 2002.

[60] A. J. Petruska, J. J. Abbott, Optimal permanent-magnet geometries for dipole field approximation, IEEE transactions on magnetics 49 (2) (2013) $811-819$. 